The AstrophysiCAL JouRnAL, 537:283-303, 2000 July 1

(c) 2000. The American Astronomical Society. All rights reserved. Printed in U.S.A.

\title{
STRUCTURE AND EVOLUTION OF THE ENVELOPES OF DEEPLY EMBEDDED MASSIVE YOUNG STARS
}

\author{
Floris F. S. VAN DER TAK AND EwINe F. VAN Dishoeck \\ Sterrewacht, Postbus 9513, 2300 RA Leiden, The Netherlands; vdtak@strw.leidenuniv.nl \\ Neal J. Evans II \\ Department of Astronomy, University of Texas, Austin, TX 78712 \\ AND \\ GeOFFrey A. BLAKE \\ Division of Geological and Planetary Sciences, California Institute of Technology, MS 150-21, Pasadena, CA 91125 \\ Received 1999 September 20; accepted 2000 January 31
}

\begin{abstract}
The physical structure of the envelopes around a sample of 14 massive young stars is investigated using maps and spectra in submillimeter continuum and lines of $\mathrm{C}^{17} \mathrm{O}, \mathrm{CS}, \mathrm{C}^{34} \mathrm{~S}$, and $\mathrm{H}_{2} \mathrm{CO}$. Nine of the sources are highly embedded luminous $\left(10^{3}-10^{5} L_{\odot}\right)$ young stellar objects that are bright nearinfrared sources but weak in radio continuum; the other objects are similar but not bright in the nearinfrared and contain "hot-core"-type objects and/or ultracompact $\mathrm{H}$ II regions. The data are used to constrain the temperature and density structure of the circumstellar envelopes on $10^{2}-10^{5} \mathrm{AU}$ scales, to investigate the relation between the different objects, and to search for evolutionary effects. The total column densities and the temperature profiles are obtained by fitting self-consistent dust models to submillimeter photometry. The calculated temperatures range from 300 to $1000 \mathrm{~K}$ at $\sim 10^{2} \mathrm{AU}$ and from 10 to $30 \mathrm{~K}$ at $\sim 10^{5} \mathrm{AU}$ from the star. Visual extinctions are a few hundred to a few thousand magnitudes, assuming a grain opacity at $1300 \mu \mathrm{m}$ of $\approx 1 \mathrm{~cm}^{-2} \mathrm{~g}^{-1}$ of dust, as derived earlier for one of our sources. The mid-infrared data are consistent with a $30 \%$ decrease of the opacity at higher temperatures, caused by the evaporation of the ice mantles. The $\mathrm{CS}, \mathrm{C}^{34} \mathrm{~S}$, and $\mathrm{H}_{2} \mathrm{CO}$ data as well as the submillimeter dust emission maps indicate density gradients $n \propto r^{-\alpha}$. Assuming a constant CS abundance throughout the envelope, values of $\alpha=1.0-1.5$ are found, which is significantly flatter than the $\alpha=2.0 \pm 0.3$ generally found for low-mass objects. This flattening may indicate that in massive young stellar objects, nonthermal pressure is more important for the support against gravitational collapse, while thermal pressure dominates for low-mass sources. We find $\alpha=2$ for two hot-core-type sources but regard this as an upper limit since, in these objects, the CS abundance may be enhanced in the warm gas close to the star. The assumption of spherical symmetry is tested by modeling infrared absorption line data of ${ }^{13} \mathrm{CO}, \mathrm{CS}$ emission-line profiles and near-infrared continuum. There is a distinct, but small deviation from spherical symmetry: the data are consistent with a decrease of the optical depth by a factor of $\approx 3$ in the central $\lesssim 10^{\prime \prime}$. The homogeneity of the envelopes is verified by the good agreement of the total masses in the power-law models with the virial masses. Modeling of $\mathrm{C}^{17} \mathrm{O}$ emission shows that $\approx 40 \%-90 \%$ of the CO is frozen out onto the dust. The $\mathrm{CO}$ abundances show a clear correlation with temperature, as expected if the abundance is controlled by freeze-out and thermal desorption. The CS abundance is $3 \times 10^{-9}$ on average, ranging from (4-8) $\times 10^{-10}$ in the cold source GL 7009S to (1-2) $\times 10^{-8}$ in the two hot-coretype sources. Dense outflowing gas is seen in the $\mathrm{CS}$ and $\mathrm{H}_{2} \mathrm{CO}$ line wings; the predominance of blueshifted emission suggests the presence of dense, optically thick material within $10^{\prime \prime}$ of the center. Interferometric continuum observations at 1300-3500 $\mu \mathrm{m}$ show compact emission, probably from a 0.3 diameter, optically thick dust component, such as a dense shell or a disk. The emission is a factor of 10-100 stronger than expected for the envelopes seen in the single-dish data, so that this component may be opaque enough to explain the asymmetric CS and $\mathrm{H}_{2} \mathrm{CO}$ line profiles. The evolution of the sources is traced by the overall temperature (measured by the far-infrared color), which increases systematically with the decreasing ratio of envelope mass to stellar mass. The observed anticorrelation of near-infrared and radio continuum emission suggests that the erosion of the envelope proceeds from the inside out. Conventional tracers of the evolution of low-mass objects do not change much over this narrow age range.
\end{abstract}

Subject headings: circumstellar matter - dust, extinction - ISM: jets and outflows ISM: molecules - stars: formation - submillimeter

\section{INTRODUCTION}

The dynamical processes governing massive star formation are much less understood than is the case for low-mass stars (Churchwell 1999; Garay \& Lizano 1999). The various observational "appearances" of low-mass star formation (T Tauri stars, FU Orionis stars, infrared Class I-III sources, molecular outflows, etc.) have been linked to a single, if rough, general evolutionary scenario (Shu et al. 1993; André, Ward-Thompson, \& Barsony 1993, 2000; Evans 1999). In contrast, no clear evolutionary sequence has been established for high-mass stars. Objects such as the $\mathrm{BN}$ object in Orion are highly embedded and emit the bulk 
of their luminosity in the mid- and far-infrared. Ultracompact (UC) $\mathrm{H}$ II regions are small $(\sim 0.1 \mathrm{pc})$ sources of freefree emission at radio wavelengths (Wood \& Churchwell 1989; Kurtz, Churchwell, \& Wood 1994). "Hot-core"-type objects have bright molecular line emission at submillimeter wavelengths, which indicates temperatures of several hundred $\mathrm{K}$ and high abundances of saturated carbonbearing molecules (e.g., Blake et al. 1987). They usually lack detectable radio emission, which might be caused by "quenching" by material accreting at rates $\gtrsim 10^{-7} M_{\odot}$ $\mathrm{yr}^{-1}$ (Walmsley 1995). The distinction between UC H II regions and hot cores is not always clear, and the two are often found located very close to each other (Cesaroni et al. 1999; Kurtz et al. 2000). As a step toward understanding the evolution of massive young stellar objects (YSOs), this paper presents models for a sample of 14 such objects.

The formation of high-mass stars is invisible at optical wavelengths because of the high opacity of the surrounding material, so that reliable age estimates for massive protostellar objects are difficult to obtain. Such estimates for massive protostars traditionally have come from the morphology of the radio continuum emission (Colley 1980), but this method applies only to relatively evolved objects. More recently, the size-to-velocity ratio or dynamical timescale of molecular outflows has been used as a measure of age, but the derived age depends strongly on the adopted dynamical model (Cabrit, Raga, \& Gueth 1997). The chemical composition of the material is a potentially powerful clock, but one that is difficult to calibrate (Charnley, Tielens, \& Millar 1992; van Dishoeck \& Blake 1998).

The density and temperature structure of the circumstellar material are also expected to change as the central star develops. For the density, power laws $n(r) \propto r^{-\alpha}$ are predicted by many theoretical models. Before collapse begins, low-mass cores may relax to a power law, with $\alpha=2.0$ for thermal support (Shu 1977) and $\alpha=1.0$ for turbulent support (Lizano \& Shu 1989; Myers \& Fuller 1992; McLaughlin \& Pudritz 1996). Once collapse begins, the density distribution tends toward $\alpha=1.5$ (Larson 1969; Shu 1977). The temperature structure $T(r)$ is determined by the luminosity, the dust opacities, and $n(r)$. Since the response of $T(r)$ to changes in luminosity is rapid, we use the observed luminosity to determine $T(r)$. Besides giving information on the dynamics of star formation, a good model of the physical structure of objects is a prerequisite for determining their chemical composition, which then by itself can be used as a powerful additional evolutionary indicator. The physical conditions around newly born massive stars also reveal some of the influence that such stars have on their surroundings, which is of interest for the effects of star formation on large ( $\gtrsim 1 \mathrm{pc})$ scales.

The physical structure of massive YSO envelopes has been studied based on dust continuum observations by, e.g., Chini, Krügel, \& Kreysa (1986), Churchwell, Wolfire, \& Wood (1990), Faison et al. (1998), and Cesaroni et al. (1999). Such studies are sensitive to the column density and the temperature of the envelope but not to density itself. A major source of uncertainty for all dust models is the choice of optical properties of the grains, which limits the accuracy of derived envelope masses to a factor of 2 (Henning 1997).

Molecular rotational lines are direct probes of the $\mathrm{H}_{2}$ density and are also sensitive to temperature. Zhou et al. (1991, 1994), and Carr et al. (1995) investigated the structure of massive star-forming regions using lines of $\mathrm{CS}, \mathrm{H}_{2} \mathrm{CO}$, and other molecules with large dipole moments. However, in these studies, the column density (or the cloud mass) was not independently constrained. Doing this requires knowledge of the molecular abundance, information that is generally unavailable except in the case of $\mathrm{CO}$, which is relatively inert and much more abundant than most molecules. Still, part of the CO may be frozen out onto grain surfaces in the cold regions far from embedded stars.

This paper uses observations of both the dust and the molecular gas to constrain the physical structure of the envelopes of a sample of massive young stars. The study builds on the method of analysis developed in a previous paper for one source, GL 2591, by van der Tak et al. (1999). The column density is inferred from continuum observations and the density from molecular lines, while the temperature is calculated self-consistently from the luminosity, dust properties, and $n(r)$. We use the derived density and temperature profiles to characterize the early evolution of massive stars and their surroundings.

\section{CHOICE OF TARGETS}

The 14 sources studied in this paper are introduced in Table 1, which lists the source names, positions, distances, luminosities, radio continuum flux densities, and associated IRAS Point Source Catalog (PSC) entries. All sources have been well studied before at radio and infrared wavelengths. They were selected to be luminous $\left(>10^{3} L_{\odot}\right)$ and visible from the Northern hemisphere. The distances to some of the sources are quite uncertain; in particular, for the sources in the Cygnus $\mathrm{X}$ region, we use a fiducial distance of $1 \mathrm{kpc}$ (van der Tak et al. 1999) for ease of scaling once better distances are available.

The main sample consists of nine deeply embedded massive young stars, which were additionally selected for mid-infrared brightness ( $>100 \mathrm{Jy}$ at $12 \mu \mathrm{m}$ ), thus allowing comparison to existing absorption-line studies. A combined analysis of emission and absorption lines gives powerful constraints on the physical structure and geometry of the system and allows a nearly complete inventory of the chemical composition of both the solid and the gas phases. These nine sources have been extensively studied in the infrared, both from the ground (Willner et al. 1982; Mitchell et al. 1990) and with the Infrared Space Observatory (ISO) (van Dishoeck et al. 1998). As a comparison sample, five luminous embedded young stars that are weak in the midinfrared and that have surroundings which contain a "hot core" and/or an ultracompact $\mathrm{H}$ II region are studied.

The full sample contains sources with luminosities between $1 \times 10^{3}$ and $2 \times 10^{5} L_{\odot}$ and distances between 0.9 and $4 \mathrm{kpc}$. The radio flux densities are at the $\sim$ mJy level for most sources, which is orders of magnitude lower than expected from an $\mathrm{H}$ in region photoionized by a star with the same luminosity. The radio emission from the bright mid-infrared sources has a spectral index $\gamma$ in the range $0.5-1.0$ and may arise in a spherical wind for which $\gamma=0.7$. The principal difference between our main sample and that of other studies of massive young stars (e.g., Tofani et al. 1995; Hunter 1998; Sridharan et al. 1999) is the selection based on mid-infrared brightness, which could introduce an orientation bias, in the sense that low-density cavities such as those produced by molecular outflows may be preferentially directed toward us. Alternatively, the high midinfrared brightness may be an evolutionary effect. Depending on the relative importance of different effects, 
TABLE 1

SOURCE SAMPLE

\begin{tabular}{|c|c|c|c|c|c|c|c|}
\hline$I R A S$ PSC & Name & $\begin{array}{l}\text { Right Ascension } \\
\text { (1950) }\end{array}$ & $\begin{array}{c}\text { Declination } \\
(1950)\end{array}$ & $\begin{array}{c}\text { Luminosity } \\
\left(L_{\odot}\right)\end{array}$ & $\begin{array}{c}\text { Distance } \\
(\mathrm{kpc})\end{array}$ & $\begin{array}{c}S_{v}(6 \mathrm{~cm}) \\
(\mathrm{mJy})\end{array}$ & References \\
\hline \multicolumn{8}{|c|}{ Bright Mid-Infrared Sources } \\
\hline $02219+6152 \ldots \ldots$ & W3 IRS 5 & 022153.1 & +615220 & $1.7 \times 10^{5}$ & 2.2 & 10.7 & $1,2,21$ \\
\hline $03236+5836 \ldots \ldots$ & GL 490 & 032338.9 & +583633 & $2.2 \times 10^{3}$ & 1 & 3.2 & $3,4,22$ \\
\hline $18117-1753 \ldots \ldots$ & W33A & 181143.7 & -175302 & $1 \times 10^{5}$ & 4 & 1.9 & 5,23 \\
\hline $18196-1331 \ldots \ldots$ & GL 2136 & 181936.6 & -133140 & $7 \times 10^{4}$ & 2 & $\ldots$ & 6 \\
\hline $18316-0602 \ldots \ldots$ & GL 7009S & 183141.6 & -060235 & $3 \times 10^{4}$ & 3 & 2.7 & 7 \\
\hline $20275+4001 \ldots \ldots$ & GL 2591 & 202735.8 & +400114 & $2 \times 10^{4}$ & 1 & 0.4 & $8,9,24$ \\
\hline $22176+6303 \ldots \ldots$ & S140 IRS 1 & 221741.08 & +630341.6 & $2 \times 10^{4}$ & 0.9 & 5.8 & $10,11,25$ \\
\hline $23116+6111 \ldots \ldots$ & NGC 7538 IRS 1 & 231136.7 & +611150.8 & $1.3 \times 10^{5}$ & 2.8 & 111 & $12,13,26$ \\
\hline $23118+6110 \ldots \ldots$ & NGC 7538 IRS 9 & 231152.8 & +611059 & $4.0 \times 10^{4}$ & 2.8 & $<0.5$ & $12,13,23$ \\
\hline \multicolumn{8}{|c|}{ Weak Mid-Infrared Sources } \\
\hline \multirow[t]{2}{*}{$20126+4104 \ldots \ldots$} & IRAS $20126+4104$ & 201241.0 & +410421 & $4.5 \times 10^{3}$ & 1 & $<0.3$ & $14,9,27$ \\
\hline & DR $21(\mathrm{OH})$ & 203714.2 & +421211 & $1 \times 10^{3}$ & 1 & $<10$ & $15,9,28$ \\
\hline $02232+6138 \ldots \ldots$ & W3 $\left(\mathrm{H}_{2} \mathrm{O}\right)$ & 022317.3 & +613858 & $2 \times 10^{4}$ & 2.2 & 1.5 & $16,2,29$ \\
\hline $17175-3544 \ldots \ldots$ & NGC 6334 IRS 1 & 171732.0 & -354405 & $1.1 \times 10^{5}$ & 1.7 & 1200 & $17,18,30$ \\
\hline $17574-2403 \ldots \ldots$ & W28 A2 (= G5.89-0.39) & 175726.8 & -240357 & $1.3 \times 10^{5}$ & 2.0 & 2100 & $19,20,31$ \\
\hline
\end{tabular}

NotE.-The first reference is for the luminosity, the second for the distance, and the third for the radio data. Spectrophotometric distances are given to one decimal place, and the corresponding luminosities are accurate to a factor of 2 . The other distances are kinematic, in which case the luminosity is uncertain by a factor of 4. The kinematic distances to W33A and GL 2136 were derived from data presented in this paper. Units of right ascension are hours, minutes, and seconds, and units of declination are degrees, arcminutes, and arcseconds.

REFERENCES.- (1) Ladd et al. 1993; (2) Humphreys 1978; (3) Chini et al. 1991; (4) Harvey et al. 1979, but see Snell et al. 1984; (5) Gürtler et al. 1991; (6) Kastner et al. 1994; (7) McCutcheon et al. 1995; (8) Lada et al. 1984; (9) Distance discussed in van der Tak et al. 1999; (10) Lester et al. 1986; (11) Crampton \& Fisher 1974; (12) Werner et al. 1979; (13) Crampton et al. 1978; (14) Cesaroni et al. 1997; (15) Chandler et al. 1993; (16) Turner \& Welch 1984; (17) Harvey \& Gatley 1983; (18) Neckel 1978; (19) Harvey et al. 1994; (20) Acord et al. 1998; (21) Tieftrunk et al. 1997; (22) Simon et al. 1983; (23) Rengarajan \& Ho 1996; (24) Campbell 1984; (25) Evans et al. 1989; (26) Pratap et al. 1992; (27) Tofani et al. 1995; (28) Johnston et al. 1984; (29) Reid et al. 1995; (30) Rodríguez et al. 1982; (31) Wood \& Churchwell 1989.

the mid-infrared bright sources in the main sample could be younger or older than the sources in the comparison sample. We will discuss this point in more detail in $\S 6.5$.

The sources were selected on isolated location within $\sim 30^{\prime \prime}$ at infrared wavelengths, so that the heating is dominated by the central star. However, while GL 2591 and GL 490 are examples of objects forming in isolation, NGC 6334, S140, NGC 7538, and W3 are regions where low-mass stars are also born, as revealed by near-infrared images (Straw \& Hyland 1989; Evans et al. 1989; Hodapp 1994; Megeath et al. 1996; Bloomer et al. 1998). In addition, S140 contains three massive objects separated by $10^{\prime \prime}-15^{\prime \prime}$, which contribute about equally to the far-infrared luminosity (Evans et al. 1989). For the other sources, this information is not available. In the case of W3 IRS 5, radio continuum observations (Claussen et al. 1994) suggest the presence of seven early B-type stars within 4", a very large number in view of the average mass function and stellar density in young clusters (Carpenter et al. 1993; Luhman et al. 1998; Alves et al. 1998). As long as the power source is much smaller than our beam, its multiplicity does not matter for our analysis.

In several cases, high-resolution continuum observations reveal the presence of a compact or ultracompact $\mathrm{H}$ II region next to the source of interest. Since luminosity and/or mass are dominated by the infrared source, our models of centrally heated sources are still reasonable approximations.

\section{OBSERVATIONS}

\subsection{Single-Dish Submillimeter Spectroscopy}

Spectroscopy of selected molecular lines in the 230, 345, and $490 \mathrm{GHz}$ bands was performed with the $15 \mathrm{~m}$ James
Clerk Maxwell Telescope (JCMT) ${ }^{1}$ in Mauna Kea, Hawaii, during various runs in 1995-1997. In total, 15-20 lines were observed per source. The antenna has an approximately Gaussian main beam of FWHM 18" at $230 \mathrm{GHz}, 14^{\prime \prime}$ at 345 $\mathrm{GHz}$, and $11^{\prime \prime}$ at $490 \mathrm{GHz}$. Detailed technical information about the JCMT and its receivers and spectrometer can be found on-line at <http://www.jach.hawaii.edu/JCMT/ home.html $>$. Receivers A2, B3i, and C2 were used as front ends at 230,345, and $490 \mathrm{GHz}$, respectively. The Digital Autocorrelation Spectrometer served as back end, with continuous calibration and natural weighting employed. Values for the main beam efficiency, $\eta_{\mathrm{mb}}$, determined by the JCMT staff from observations of Mars and Jupiter, were $0.69,0.58$, and 0.53 at $230 ; 345$ and $490 \mathrm{GHz}$ for the 1995 data; and 0.64, 0.60, and 0.53 for 1996 and 1997. Absolute calibration should be correct to $30 \%$, except for data in the $230 \mathrm{GHz}$ band from May 1996, which have an uncertainty of $\approx 50 \%$ because of technical problems with receiver $\mathrm{A} 2$. Pointing was checked every $2 \mathrm{hr}$ during the observing and was always found to be within $5^{\prime \prime}$. Integration times are 30-40 minutes per frequency setting, resulting in rms noise levels in $T_{\mathrm{mb}}$ per $625 \mathrm{kHz}$ channel ranging from $\approx 30 \mathrm{mK}$ at $230 \mathrm{GHz}$ to $\approx 100 \mathrm{mK}$ at $490 \mathrm{GHz}$.

To subtract the atmospheric and instrumental background, a reference position $180^{\prime \prime}$ east was observed using the chopping secondary mirror. For the $\mathrm{C}^{17} \mathrm{O} J=2 \rightarrow 1$ line, we also position-switched using an $1800^{\prime \prime}$ offset, which increased the line fluxes typically by $15 \%$. The contribution

\footnotetext{
${ }^{1}$ The James Clerk Maxwell Telescope is operated by the Joint Astronomy Centre, on behalf of the Particle Physics and Astronomy Research Council of the United Kingdom, the Netherlands Organization for Scientific Research, and the National Research Council of Canada.
} 
by extended emission in other lines should be less since they all need higher densities for the excitation. Where two measurements of $\mathrm{C}^{17} \mathrm{O} J=2 \rightarrow 1$ are available, the positionswitched data will be used.

Observations of the $\mathrm{C}^{34} \mathrm{~S} J=2 \rightarrow 1$ and $J=3 \rightarrow 2$ and CS $J=5 \rightarrow 4$ lines were carried out with the $30 \mathrm{~m}$ telescope of the Institut de Radio Astronomie Millimétrique (IRAM) on Pico Veleta, Spain, on 1999 January 28-30. Receivers B100, C150, and B230 were used at 96, 145, and $245 \mathrm{GHz}$, respectively, tuned single sideband. The autocorrelator was used as the back end, which covers a bandwidth of 110-170 $\mathrm{km} \mathrm{s}^{-1}$ at a resolution of $0.2 \mathrm{~km} \mathrm{~s}^{-1}$. The FWHM beam sizes are $24^{\prime \prime}, 16^{\prime \prime}$, and 10".4; the forward efficiencies were $0.90,0.82$, and 0.84 and the beam efficiencies were $0.75,0.55$, and 0.48 . The data were calibrated onto the $T_{\mathrm{mb}}$ scale by multiplying $T_{A}^{*}$ by the ratio of the forward and beam efficiencies. As a calibration check, observations of GL 2591 and NGC 7538 IRS 1 were compared to results from Plume et al. (1997) and found to agree to $10 \%$.

The data on GL 2591 have been presented in van der Tak et al. (1999). The observations on GL 490 are given in K. Schreyer et al. (2000, in preparation). Most of the data on W3 $\left(\mathrm{H}_{2} \mathrm{O}\right)$ and W3 IRS 5 are taken from Helmich \& van Dishoeck (1997). In addition, we used data from the surveys by Anglada et al. (1996), Bronfman, Nyman, \& May (1996),
Plume, Jaffe, \& Evans (1992, 1997), and from the observations of Kastner et al. (1994) for GL 2136; Zhou et al. (1994) for S140 IRS 1; Cesaroni et al. (1997) for IRAS 20126; Hauschildt et al. (1993) and Mangum \& Wootten (1993) for DR $21(\mathrm{OH})$; Dartois (1998) for GL 7009S; and Olmi \& Cesaroni (1999) for W28 A2. Care was taken not to include data at positions greater than $5^{\prime \prime}$ away from those in Table 1. For the sources GL 7009S, IRAS 20126+4104, and W28 A2, no $\mathrm{H}_{2} \mathrm{CO}$ data are available.

Line parameters were measured by fitting a Gaussian profile, where the free parameters were the total line flux $\int T_{\mathrm{MB}} d V$, the FWHM line width, and the center velocity. The measured line fluxes are collected in Table 2; Table 3 gives for each source the central LSR velocity and the FWHM line width. The values are the averages of the $\mathrm{C}^{17} \mathrm{O}$ and $\mathrm{C}^{34} \mathrm{~S}$ lines (four lines in most cases), which are assumed to be optically thin. The error bars reflect the spread among the lines, which for all sources exceeds the error in the individual measurements for both quantities. None of the CS and $\mathrm{H}_{2} \mathrm{CO}$ line profiles show obvious evidence of selfabsorption.

\subsection{Single-Dish Mapping}

Maps of the CS $J=5 \rightarrow 4$ and/or $7 \rightarrow 6$ and/or $\mathrm{C}^{18} \mathrm{O}$ $J=2 \rightarrow 1$ lines were obtained in 1998 July and December

TABLE 2

OBSERVED Line FluXes $\int T_{\mathrm{mb}} d V\left(\mathrm{~K} \mathrm{~km} \mathrm{~s}^{-1}\right)$

\begin{tabular}{|c|c|c|c|c|c|c|c|c|c|}
\hline Line & $\begin{array}{c}\text { W3 } \\
\text { IRS } 5\end{array}$ & GL 490 & W33A & GL 2136 & $\begin{array}{c}\text { S140 } \\
\text { IRS } 1\end{array}$ & $\begin{array}{c}\text { NGC } 7538 \\
\text { IRS } 1\end{array}$ & $\begin{array}{c}\text { NGC } 7538 \\
\text { IRS } 9\end{array}$ & $\begin{array}{c}\text { NGC } 6334 \\
\text { IRS } 1\end{array}$ & $\begin{array}{c}\mathrm{W} 3 \\
\left(\mathrm{H}_{2} \mathrm{O}\right)\end{array}$ \\
\hline \multicolumn{10}{|c|}{ JCMT Observations } \\
\hline $\mathrm{C}^{17} \mathrm{O} 2-1^{\mathrm{a}} \ldots \ldots \ldots \ldots \ldots$ & 12.2 & $\ldots$ & 11.3 & 6.8 & 6.4 & 8.7 & 3.8 & 26.5 & $\ldots$ \\
\hline $\mathrm{C}^{17} \mathrm{O} 3-2 \ldots \ldots \ldots \ldots \ldots$ & 16.6 & 4.9 & 15.7 & 9.9 & 10.8 & 17.9 & 4.5 & 57.9 & 15.1 \\
\hline CS $5-4 \ldots \ldots \ldots \ldots \ldots \ldots$ & $\ldots$ & 16.5 & 36.2 & 11.2 & 30.2 & 54.8 & 20.6 & $\ldots$ & $\ldots$ \\
\hline CS $7-6 \ldots \ldots \ldots \ldots \ldots \ldots$ & 25.2 & 8.0 & 17.4 & 4.7 & 22.3 & 56.0 & 20.5 & 162. & 83.7 \\
\hline CS $10-9 \ldots \ldots \ldots \ldots \ldots$ & 16.1 & $\ldots$ & $\ldots$ & $\ldots$ & $\ldots$ & 34.4 & 3.3 & $\ldots$ & 78.4 \\
\hline $\mathrm{C}^{34} \mathrm{~S} 5-4^{\mathrm{b}} \ldots \ldots \ldots \ldots \ldots$ & 3.7 & 1.1 & 5.6 & 1.1 & 2.7 & 7.0 & 2.4 & 56.5 & 20.7 \\
\hline 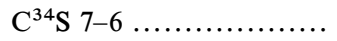 & 2.5 & 0.6 & $5.3^{\mathrm{d}}$ & $<0.4$ & 2.1 & $7.9^{\mathrm{d}}$ & 3.1 & $64.8^{\mathrm{d}}$ & 25.8 \\
\hline$C^{34} S 10-9 \ldots \ldots \ldots \ldots \ldots$ & 2.2 & $\ldots$ & $\ldots$ & $\ldots$ & $\ldots$ & 4.6 & $<3.4$ & $\ldots$ & 31.6 \\
\hline $\mathrm{H}_{2} \mathrm{CO} 3_{03}-2_{02} \ldots \ldots \ldots \ldots$ & 8.5 & 4.4 & 13.1 & 4.4 & 13.7 & 27.0 & 14.2 & 63.2 & 20.2 \\
\hline $\mathrm{H}_{2} \mathrm{CO} 3_{12}-2_{11} \ldots \ldots \ldots \ldots$ & 19.0 & $\ldots$ & 15.0 & 6.3 & 19.2 & 27.0 & 13.6 & 63.0 & 41.2 \\
\hline $\mathrm{H}_{2} \mathrm{CO} 3_{22}-2_{21} \ldots \ldots \ldots \ldots$ & 3.6 & 0.7 & 3.5 & 1.6 & 3.5 & 7.1 & 3.7 & 26.2 & 6.9 \\
\hline $\mathrm{H}_{2} \mathrm{CO} 5_{05}-4_{04} \ldots \ldots \ldots \ldots$ & 5.2 & $\ldots$ & 10.9 & 4.3 & 8.7 & 18.2 & 9.5 & 82.9 & 31.1 \\
\hline 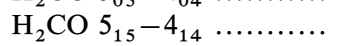 & 14.0 & $\ldots$ & 20.0 & 7.4 & 20.5 & 28.8 & $\ldots$ & 105. & 36.2 \\
\hline $\mathrm{H}_{2} \mathrm{CO} 5_{24}-4_{23} \ldots \ldots \ldots \ldots$ & 2.7 & 1.6 & 4.5 & 2.2 & 3.7 & 7.9 & 4.5 & 52.9 & 19.5 \\
\hline $\mathrm{H}_{2} \mathrm{CO} 5_{32}-4_{31} \ldots \ldots \ldots \ldots$ & 2.9 & 1.5 & 5.4 & 1.5 & 3.6 & 8.8 & 4.5 & 48.1 & $33.4^{\mathrm{e}}$ \\
\hline $\mathrm{H}_{2} \mathrm{CO} 5_{33}-4_{32} \ldots \ldots \ldots \ldots$ & 2.6 & 1.2 & 4.9 & 2.6 & 3.3 & 9.9 & 4.2 & 50.6 & 24.7 \\
\hline $\mathrm{H}_{2} \mathrm{CO} 5_{42 / 41}-4_{41 / 40} \cdots \cdots$ & 0.9 & $<0.7$ & 1.5 & $<0.4$ & $<0.5$ & 2.4 & 1.3 & 29.4 & 8.3 \\
\hline $\mathrm{H}_{2} \mathrm{CO} 7_{17}-6_{16} \ldots \ldots \ldots \ldots$ & 21.9 & $\ldots$ & $\ldots$ & $\ldots$ & 7.3 & 15.8 & 10.8 & $\ldots$ & 24.3 \\
\hline \multicolumn{10}{|c|}{ IRAM $30 \mathrm{~m}$ Observations } \\
\hline CS $5-4 \ldots \ldots \ldots \ldots \ldots \ldots \ldots$ & $\ldots$ & $\ldots$ & 80.7 & 22.5 & 37.7 & 79.0 & 37.2 & $\ldots$ & $\ldots$ \\
\hline $\mathrm{C}^{34} \mathrm{~S} 2-1 \ldots \ldots \ldots \ldots \ldots$ & $\ldots$ & $\ldots$ & 7.6 & 1.8 & 3.6 & $\ldots$ & 4.1 & $\ldots$ & $\ldots$ \\
\hline $\mathrm{C}^{34} \mathrm{~S} 3-2 \ldots \ldots \ldots \ldots \ldots$ & $\ldots$ & $\ldots$ & $\ldots$ & 2.4 & 4.3 & $\ldots$ & 5.4 & $\cdots$ & $\cdots$ \\
\hline \multicolumn{10}{|c|}{ CSO Observations } \\
\hline $\mathrm{CS} 5-4^{\mathrm{c}} \ldots \ldots \ldots \ldots \ldots \ldots$ & 21.5 & $\ldots$ & 20.3 & 8.3 & 30.5 & 54.4 & 18.2 & 78.6 & 33.5 \\
\hline CS $7-6 \ldots \ldots \ldots \ldots \ldots \ldots$ & 20.0 & 4.9 & 10.5 & 6.6 & 20.7 & 50.7 & $<0.2$ & 103.6 & 64.2 \\
\hline $\mathrm{C}^{34} \mathrm{~S} 7-6 \ldots \ldots \ldots \ldots \ldots$ & $\ldots$ & $\ldots$ & $\ldots$ & $\ldots$ & $\ldots$ & $\ldots$ & $\ldots$ & $\ldots$ & 15.8 \\
\hline $\begin{array}{l}{ }^{\text {a }} \text { For this line, a flux of } 2.9 \\
{ }^{b} \text { For this line, a flux of } 0.5 \\
{ }^{\mathrm{c}} \text { For this line, a flux of les } \\
(\mathrm{OH}) \text {, and } 123.2 \mathrm{~K} \mathrm{~km} \mathrm{~s}^{-1} \text { fo } \\
{ }_{\mathrm{d}} \text { Blend with triplet of CN } \\
{ }^{\mathrm{e}} \text { Blend with } \mathrm{CH}_{3} \mathrm{OCHO}\end{array}$ & $\begin{array}{l}\mathrm{km} \mathrm{s}^{-1} \\
\mathrm{~km} \mathrm{~s}^{-1} \\
\operatorname{lan} 0.8 \\
28 \mathrm{~A} 2 \\
\text { es; fit co }\end{array}$ & $\begin{array}{l}\text { s measure } \\
\text { s measure } \\
\mathrm{m} \mathrm{s}^{-1} \text { wa } \\
\text { rained by } \\
\text { it constre }\end{array}$ & $\begin{array}{l}\text { r IRA } \\
\text { r GL } \\
\text { easure } \\
\text { uiring }\end{array}$ & $\begin{array}{l}126 \text { and } 24 \\
9 \mathrm{~S}, 3.2 \mathrm{~K} \mathrm{kn} \\
\mathrm{r} \mathrm{GL} 7009 \\
\text { ne } \Delta V \text { as ot } \\
\text { ring same } \Delta\end{array}$ & $\begin{array}{l}\mathrm{K} \mathrm{km} \mathrm{s} \\
-1 \text { for II } \\
\text { f less th }\end{array}$ & $\begin{array}{l}\mathrm{r} \text { DR } 21(\mathrm{O}) \\
20126 \text {, and } \\
6 \mathrm{~K} \mathrm{~km} \mathrm{~s} \\
\mathrm{C}^{17} \mathrm{O} \text { lines. } \\
\mathrm{CO} \text { lines. }\end{array}$ & $\begin{array}{l}9 \mathrm{~K} \mathrm{~km} \mathrm{~s}^{-1} \\
\mathrm{r} \text { IRAS } 2012\end{array}$ & $\begin{array}{l}\mathrm{W} 28 \mathrm{~A} 2 \text {. } \\
9.7 \mathrm{~K} \mathrm{~km} \mathrm{~s}\end{array}$ & r DR 21 \\
\hline
\end{tabular}


TABLE 3

Single-Dish Line Emission: Basic Parameters

\begin{tabular}{|c|c|c|c|c|}
\hline Source & $\begin{array}{c}V_{\mathrm{LSR}} \\
\left(\mathrm{km} \mathrm{s}^{-1}\right)\end{array}$ & $\begin{array}{c}\Delta V \\
\left(\mathrm{~km} \mathrm{~s}^{-1}\right)\end{array}$ & $\begin{array}{c}N(\mathrm{CO}) \\
\left(10^{19} \mathrm{~cm}^{-2}\right)\end{array}$ & $\begin{array}{c}D(\mathrm{CS}) \\
(\operatorname{arcsec})\end{array}$ \\
\hline W3 IRS $5 \ldots \ldots \ldots \ldots$ & $-38.4(0.3)$ & $2.7(1.1)$ & 3.7 & 55 \\
\hline GL $490 \ldots \ldots \ldots \ldots \ldots$ & $-13.3(0.2)$ & $3.3(0.6)$ & 0.8 & 38 \\
\hline W33A.................. & $+37.5(0.9)$ & $5.4(0.5)$ & 3.1 & 37 \\
\hline GL $2136 \ldots \ldots \ldots \ldots \ldots$ & $+22.8(0.1)$ & $3.1(0.4)$ & 1.4 & 36 \\
\hline GL 7009S $\ldots . . \ldots \ldots \ldots$ & $+40.3(0.6)$ & $5.0(1.0)$ & $\ldots$ & $\ldots$ \\
\hline GL $2591^{a} \ldots \ldots \ldots \ldots \ldots$ & $-5.5(0.2)$ & $3.3(0.6)$ & 3.4 & 52 \\
\hline S140 IRS $1 \ldots \ldots \ldots \ldots$ & $-7.0(0.2)$ & $3.3(0.3)$ & 1.4 & 64 \\
\hline NGC 7538 IRS $1 \ldots \ldots$ & $-57.4(0.5)$ & $4.1(1.4)$ & 3.9 & 52 \\
\hline NGC 7538 IRS $9 \ldots \ldots$ & $-57.2(0.3)$ & $4.1(0.5)$ & 1.0 & 47 \\
\hline IRAS $20126^{b} \ldots \ldots \ldots \ldots$ & $-3.7(0.2)$ & $3.2(0.2)$ & 0.8 & 40 \\
\hline DR $21(\mathrm{OH}) \ldots \ldots \ldots \ldots$ & $-3.1(0.2)$ & $4.5(0.2)$ & 15.6 & 56 \\
\hline $\mathrm{W} 3\left(\mathrm{H}_{2} \mathrm{O}\right) \ldots \ldots \ldots \ldots \ldots$ & $-47.6(0.6)$ & $5.8(0.6)$ & 8.5 & 41 \\
\hline NGC 6334 IRS $1 \ldots .$. & $-7.4(0.2)$ & $5.3(0.3)$ & 16.9 & 50 \\
\hline $\mathrm{W} 28 \mathrm{~A} 2^{\mathrm{c}} \ldots \ldots \ldots \ldots \ldots$ & +10 & 5.7 & $\ldots$ & 38 \\
\hline
\end{tabular}

Note- The $\mathrm{CO}$ column densities in fourth column are derived from $\mathrm{C}^{17} \mathrm{O}$ emission assuming ${ }^{16} \mathrm{O} /{ }^{17} \mathrm{O}=2500$, refer to a $14^{\prime \prime}$ beam and have a $30 \%$ calibration uncertainty. The last column lists the half-power diameters of the CS $J=5 \rightarrow 4$ emission mapped with the CSO.

${ }^{\text {a }}$ Size of CS emission from data presented in Carr et al. 1995.

${ }^{b}$ Size of CS emission from data presented in Cesaroni et al. 1997.

' Velocity and line width taken from Plume et al. 1997.

and 1999 July with the $10.4 \mathrm{~m}$ antenna of the Caltech Submillimeter Observatory $(\mathrm{CSO}){ }^{2}$ The back ends were the facility 500 and $50 \mathrm{MHz}$ bandwidth Acousto-Optical Spectrometers (AOS). The pointing is accurate to within 4". Beam sizes and main beam efficiencies were $32^{\prime \prime}$ and 0.66 at $230 \mathrm{GHz}$ and $21^{\prime \prime}$ and 0.61 at $345 \mathrm{GHz}$.

In July 1998, submillimeter continuum maps at $350 \mu \mathrm{m}$ of W33A, GL 2136, S140, GL 490, GL 2591, and W3 IRS 5 were made using the Submillimeter High Angular Resolution Camera (SHARC) (Hunter, Benford, \& Serabyn 1996) on the CSO. The CSO has a beam size of $10^{\prime \prime}$ at this wavelength. The weather was good, with zenith optical depths at $350 \mu \mathrm{m}$ of $0.92-1.66$. From observations of Uranus, the gain was measured to be $(140 \pm 30) \mathrm{Jy} \mathrm{V}^{-1}$.

\subsection{Interferometer Observations}

Maps at $86-230 \mathrm{GHz}$ of GL 2136, NGC 7538 IRS 1, NGC 7538 IRS 9, and W33A were obtained with the sixelement interferometer of the Owens Valley Radio Observatory (OVRO). ${ }^{3}$ The OVRO interferometer consists of six $10.4 \mathrm{~m}$ antennas on north-south and east-west baselines. A detailed technical description of the instrument is given in Padin et al. (1991). Data were collected in 1997-1999 in several array configurations at frequencies of 86,106 , and $115 \mathrm{GHz}$. The two sources in NGC 7538 were observed in the compact and extended array configurations, while for the southern sources GL 2136 and W33A, a hybrid configuration with long north-south and short east-west baselines was also used to improve the beam shape. Baseline lengths range from the shadowing limit out to $\sim 80 \mathrm{k} \lambda$ at $86 \mathrm{GHz}$ and out to $150 \mathrm{k} \lambda$ at $230 \mathrm{GHz}$, corresponding to spatial frequencies of $\sim 2500$ to $\sim 10^{5} \mathrm{rad}^{-1}$.

\footnotetext{
${ }^{2}$ The Caltech Submillimeter Observatory is operated by the California Institute of Technology under funding from the US National Science Foundation (AST96-15025).

${ }^{3}$ The Owens Valley Millimeter Array is operated by the California Institute of Technology under funding from the US National Science Foundation (AST96-13717).
}

The antenna gains and phases were monitored with short integrations of nearby quasars: BL Lac for NGC 7538 IRS 1 and IRS 9, and NRAO 530 for W33A and GL 2136. Passband corrections were derived using a local signal generator and by fitting first-order polynomials to data on 3C273. Flux calibration is based on snapshots of Uranus and Neptune and is estimated to be accurate to $\approx 30 \%$ at $86-115 \mathrm{GHz}$ and to $\approx 50 \%$ at $230 \mathrm{GHz}$.

Simultaneous continuum observations in the $230 \mathrm{GHz}$ window were also performed, but only in the cases of W33A and NGC 7538 IRS 1 did the weather allow usable data to be taken. In addition, observations of molecular lines at 86-230 GHz were carried out with the OVRO digital correlator, the results of which will be presented elsewhere.

\section{RESULTS}

\subsection{Molecular Emission-Line Profiles}

The CS line profiles are presented in Figure 1. In addition to a strong, single-peaked line core, which is also detected in isotopic lines $\left(\mathrm{C}^{34} \mathrm{~S}, \mathrm{C}^{17} \mathrm{O}\right)$ and which has an approximately Gaussian shape, high-velocity wings are clearly detected. These wings are more prominent in $J=5 \rightarrow 4$ than in higher $J$ lines, are also detected in the strongest $\mathrm{H}_{2} \mathrm{CO}$ lines, and must arise on small scales since they are stronger in the IRAM $30 \mathrm{~m}$ beam than in the JCMT beam. The wings are not detected in the W3 sources, for which we do not have $J=5 \rightarrow 4$ data. Since the sources studied in this paper are known to drive $\mathrm{CO}$ outflows, it is natural to associate the $\mathrm{CS}$ and $\mathrm{H}_{2} \mathrm{CO}$ wings with dense gas in these outflows.

In all bright mid-infrared sources studied in this paper except W33A, blueshifted infrared absorption of CO is detected at similar velocities as in $\mathrm{CO}$ rotational line emission (Mitchell, Maillard, \& Hasegawa 1991), and in the cases of W3 IRS 5 and GL 2591, up to much higher velocities, implying that the outflow is directed along the line of sight. In realistic outflow models with a finite opening angle, a mixture of blue- and redshifted emission is expected on both sides of the driving source, which should be visible in 


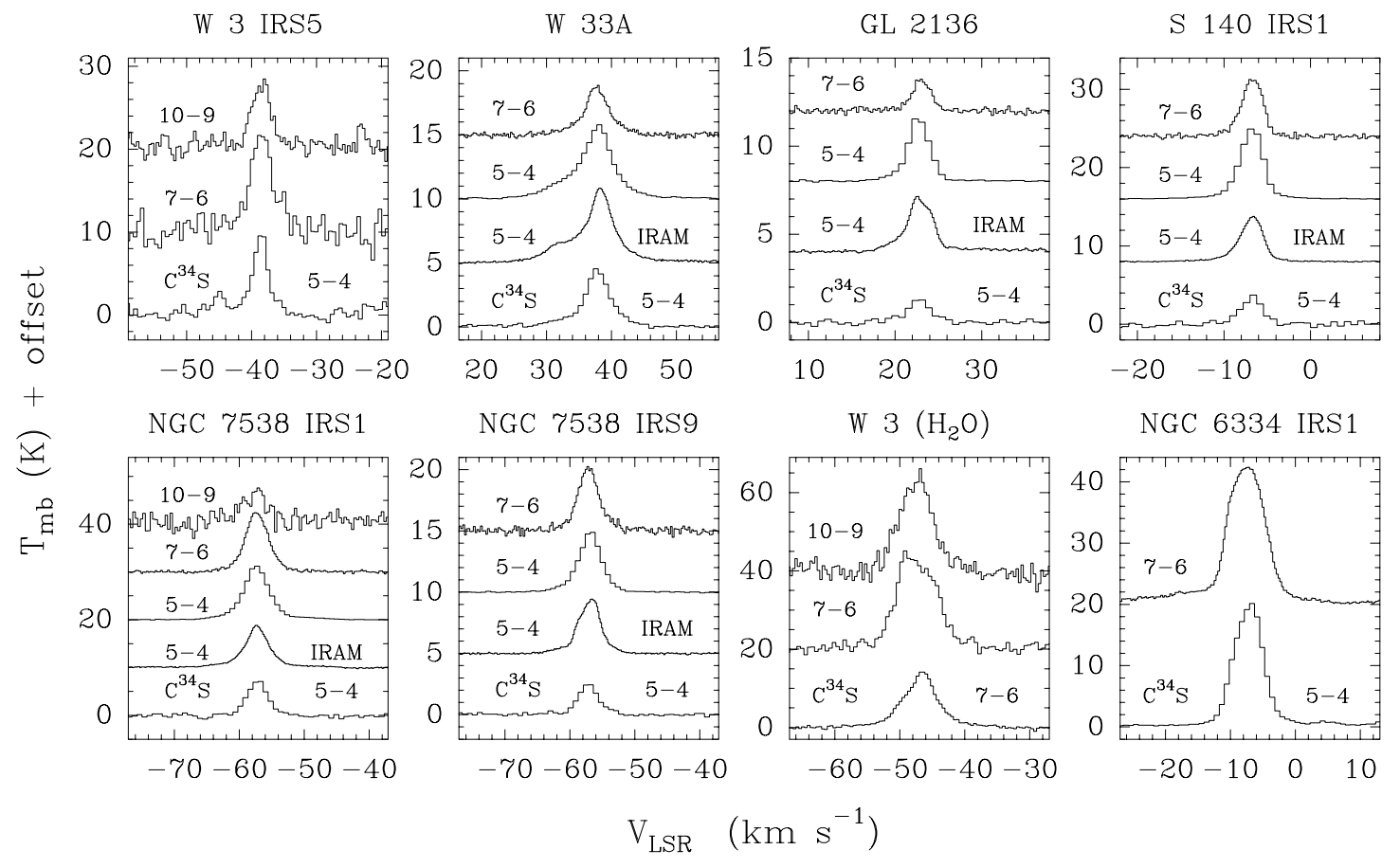

FIG. 1.- Line profiles of CS and $\mathrm{C}^{34} \mathrm{~S}$, observed with the JCMT and IRAM $30 \mathrm{~m}$ telescopes. For clarity, the IRAM data have been divided by 2 and the $\mathrm{C}^{34} \mathrm{~S}$ data have been multiplied by 2 for the hot cores and by 3 for the other sources. At blueshifted velocities, wings are visible on the CS lines, most prominently in the IRAM data.

submillimeter emission. However, the wings seen on the CS and $\mathrm{H}_{2} \mathrm{CO}$ lines are much stronger at blueshifted than at redshifted velocities, and for some sources no redshifted wing emission is detected at all. Since the line asymmetry is stronger in the IRAM $30 \mathrm{~m}$ spectrum of CS $J=5 \rightarrow 4$ than in the JCMT profile of the same line, the asymmetry must arise on scales of $\lesssim 10^{\prime \prime}$. Since the redshifted outflow lobe lies in the background, we suggest that the lack of redshifted $\mathrm{CO}$ and CS wing emission is caused by obscuration. Since the outflow lies at a velocity offset, the absorption must be continuous absorption by dust. The $\mathrm{H}_{2}$ column density required to do this is $\sim 10^{25} \mathrm{~cm}^{-2}$ on a $\lesssim 10^{\prime \prime}$ scale, corresponding to a visual extinction of $\sim 10^{4}$ magnitudes.

\subsection{Average Physical Conditions}

Estimates of the mean temperature and density of the gas can be obtained by comparing observed line ratios of specific molecules with non-LTE models that include radiative trapping. Examples of this approach can be found in Jansen, van Dishoeck, \& Black (1994) and Helmich et al. (1994); it is similar to the "Large Velocity Gradient" method used by Zhou et al. (1994) and Plume et al. (1997). The observed line ratios of $\mathrm{C}^{34} \mathrm{~S}$ are well suited to constrain the $\mathrm{H}_{2}$ density. We have calculated synthetic line ratios for a range of temperatures and densities using the rate coefficients by S. Green (1992, private communication, cf. Turner et al. 1992). Comparison with the data indicates densities of $10^{5}$ to greater than $10^{7} \mathrm{~cm}^{-3}$, but different line ratios generally give inconsistent answers for the same source, indicating the presence of density inhomogeneities, probably in the form of a gradient since lines observed with several telescopes are systematically brighter when observed with a smaller beam.

The measured ratios of the $\mathrm{H}_{2} \mathrm{CO} J=5_{24} \rightarrow 4_{23} / J=$ $5_{05} \rightarrow 4_{04}$ and the $J=5_{42 / 41} \rightarrow 4_{41 / 40} / J=5_{24} \rightarrow 4_{23}$ lines are particularly sensitive to temperature. Unlike $\mathrm{C}^{34} \mathrm{~S}$, these
$\mathrm{H}_{2} \mathrm{CO}$ line ratios were measured in the same beam, or even in the same spectrum, improving the calibration between pairs of lines, although their filling factors still might be different. Model calculations using collisional rate coefficients by Green (1991) give temperatures of $\approx 60 \mathrm{~K}$ for $\mathrm{S} 140$ IRS 1 to greater than $200 \mathrm{~K}$ for W3 $\left(\mathrm{H}_{2} \mathrm{O}\right)$ and NGC 6334 IRS 1 . Again, the two ratios usually do not yield the same temperature for the same source, indicating the need for models with a varying temperature. In general, the modeling indicates somewhat higher temperatures and densities for the weak mid-infrared sources. In $\S 5$, we will see that this effect is caused by a steeper density gradient in these sources.

The CO column densities derived from $\mathrm{C}^{17} \mathrm{O}$, using the temperatures and densities found above, are listed in the third column of Table 3. A plot of these values against the column densities derived by Mitchell et al. (1990) from infrared absorption observations is shown in Figure 2. Abundance ratios of ${ }^{12} \mathrm{C} /{ }^{13} \mathrm{C}=60$ and ${ }^{16} \mathrm{O} /{ }^{17} \mathrm{O}=2500$ (Wilson \& Rood 1994) are assumed. The two measurements agree to a factor of 2 for all sources, and often have much better agreement. This result implies that the circumstellar envelopes of our objects have to first order a spherical shape, with the infrared source in the center (see also § 6.1). The average absorption column density is $66 \%$ of the emission value, which is higher than half as expected in a uniform model. Beam dilution in the emission data could explain this difference, in which case the sources are centrally condensed.

\subsection{Submillimeter Continuum and Line Maps}

The $\mathrm{CS}$ and $\mathrm{C}^{18} \mathrm{O}$ maps are presented in Figure 3; Figure 4 shows the SHARC $350 \mu \mathrm{m}$ maps and maps of NGC 7538 at 450 and $850 \mu \mathrm{m}$ obtained with the Submillimeter Common-User Bolometer Array (SCUBA) on the JCMT and provided by G. Sandell (1998, private communication). 


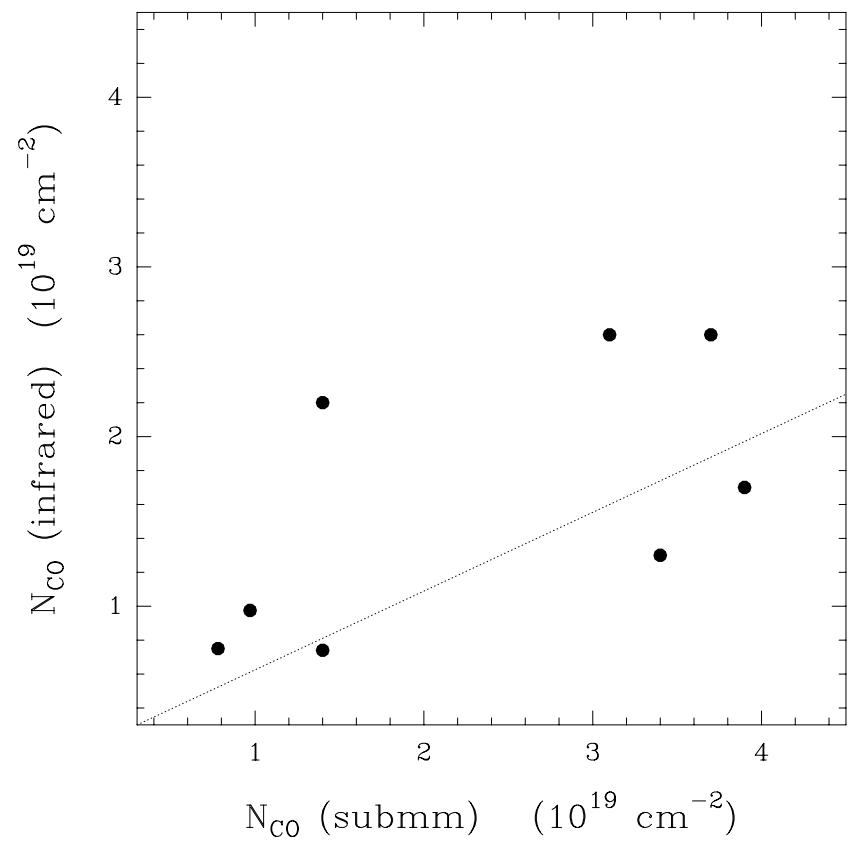

FIG. 2.-Column densities of $\mathrm{CO}$ derived from $\mathrm{C}^{17} \mathrm{O}$ emission compared to values derived from infrared absorption of ${ }^{13} \mathrm{CO}$. The dotted line indicates the relation expected for a constant-density model.

The maps appear compact but slightly extended. In a few cases, the map peak is offset from the center position, but this offset falls within the $5^{\prime \prime}$ pointing uncertainty. In the case of CS, this suggests that the infrared sources coincide with local density maxima in the surrounding molecular cloud. The $\mathrm{C}^{18} \mathrm{O}$ maps are sometimes peaked at the same position as CS, which implies a maximum of the column density, but more often, these maps are not strongly peaked and have a much lower dynamic range than the CS maps. The map of NGC 7538 shows a chaotic structure rather than clear peaks.

The fourth column of Table 3 lists the diameter of the CS $J=5 \rightarrow 4$ emission measured from the maps as the point where the brightness has dropped to $50 \%$ of the maximum. These numbers will be used in the next section to constrain the radii of the models. The values span a fairly narrow range, $36^{\prime \prime}-64^{\prime \prime}$, and do not show an obvious correlation with distance, suggesting that the cores do not have a single intrinsic linear size. Instead, it appears that the molecular gas has a scale-free density structure, such as can be described by a power law. This conclusion is supported by the fact that the CS diameters are somewhat $(10 \%-100 \%)$ larger than the beam size, which is also what power-law models predict.

The maps do not show a cutoff in the emission, such as would be produced if the star-forming cores had a distinct edge. Rather, the brightness keeps going down until the detection limit. Maps of a wider field and with a higher dynamic range than presented here may reveal if there is such an edge or if at large radii the density gradient flattens out and the core merges into a surrounding molecular cloud. Some objects, such as W3 IRS 5, DR $21(\mathrm{OH})$, NGC 6334 IRS 1, and the NGC 7538 sources, are clearly part of a more extended giant molecular cloud. However, the focus of this paper is on the $\sim 30^{\prime \prime}$ region around the massive young stars.

\subsection{Interferometric Continuum Maps}

Figure 5 presents maps created from the OVRO data by gridding and Fourier transforming the visibility data with uniform weighting. Deconvolution with the CLEAN algorithm and self-calibration of the $u-v$ phases based on the brightest CLEAN components helped to improve the image quality. Table 4 lists the positions and flux densities of the detected sources.

The maps typically show a single compact source at or near phase center, within the $\sim 5^{\prime \prime}$ positional error of the infrared positions from Table 1 . The extended $107 \mathrm{GHz}$ emission north of NGC 7538 IRS 1 is the H II region surrounding NGC 7538 IRS 2, which has also been detected at lower frequencies with the Very Large Array (VLA) (e.g., Henkel, Wilson, \& Johnston 1984). In the case of W33A, two sources are detected, neither of which coincides with the IR position of Dyck \& Simon (1977). The brightest source, MM 1, however, coincides with a VLA detection (Rengarajan \& Ho 1996) and with the infrared position by Capps, Gillett, \& Knacke (1978), who also report a second $2.2 \mu \mathrm{m}$ source $3^{\prime \prime}$ south of W33A, which may be related to MM 2. It is unknown if these two sources are physically associated; if they are, they may represent a young massive

TABLE 4

RESULTS OF OVRO OBSERVATIONS

\begin{tabular}{|c|c|c|c|c|c|}
\hline Source & $\begin{array}{l}\text { Frequency } \\
(\mathrm{GHz})\end{array}$ & R.A. (1950) & Decl. (1950) & $\begin{array}{l}\text { Flux Density } \\
(\mathrm{mJy})\end{array}$ & $\begin{array}{c}\text { Beam Size } \\
(\operatorname{arcsec})\end{array}$ \\
\hline GL $2136 \ldots . .$. & 86 & $181936.62(0.02)$ & $-133143.8(0.3)$ & 61 & $3.0 \times 2.8$ \\
\hline \multirow{3}{*}{ W33A MM $1 \ldots \ldots \ldots}$. & 86 & $181144.22(0.02)$ & $-175258.2(0.2)$ & 24 & $3.3 \times 2.7$ \\
\hline & 106 & $\ldots$ & $\ldots$ & 54 & $2.9 \times 2.6$ \\
\hline & 233 & $\ldots$ & $\ldots$ & 190 & $2.0 \times 1.6$ \\
\hline \multirow[t]{3}{*}{ W33A MM $2 \ldots \ldots \ldots}$. & 86 & $181143.91(0.02)$ & $-175259.8(0.2)$ & 38 & $3.3 \times 2.7$ \\
\hline & 106 & $\ldots$ & $\ldots$ & 30 & $2.9 \times 2.6$ \\
\hline & 233 & $\ldots$ & $\ldots$ & 140 & $2.0 \times 1.6$ \\
\hline \multirow[t]{4}{*}{ NGC 7538 IRS $1 \ldots \ldots$} & 86 & $231136.64(0.02)$ & $+611149.8(0.2)$ & 1500 & $2.6 \times 2.0$ \\
\hline & 107 & $\ldots$ & $\ldots$ & 1800 & $2.4 \times 1.9$ \\
\hline & 115 & $\ldots$ & $\ldots$ & 2000 & $2.5 \times 1.7$ \\
\hline & 230 & $\ldots$ & $\ldots$ & 3500 & $1.1 \times 0.9$ \\
\hline \multirow[t]{2}{*}{ NGC 7538 IRS $9 . . .}$. & 107 & $231152.90(0.07)$ & $+611059.2(0.5)$ & 43 & $2.4 \times 2.0$ \\
\hline & 115 & $\ldots$ & $\ldots$ & 95 & $2.4 \times 1.9$ \\
\hline
\end{tabular}

NoTE-Units of right ascension are hours, minutes, and seconds, and units of declination are degrees, arcminutes, and arcseconds. The flux densities have a calibration uncertainty of $\approx 30 \%$. 

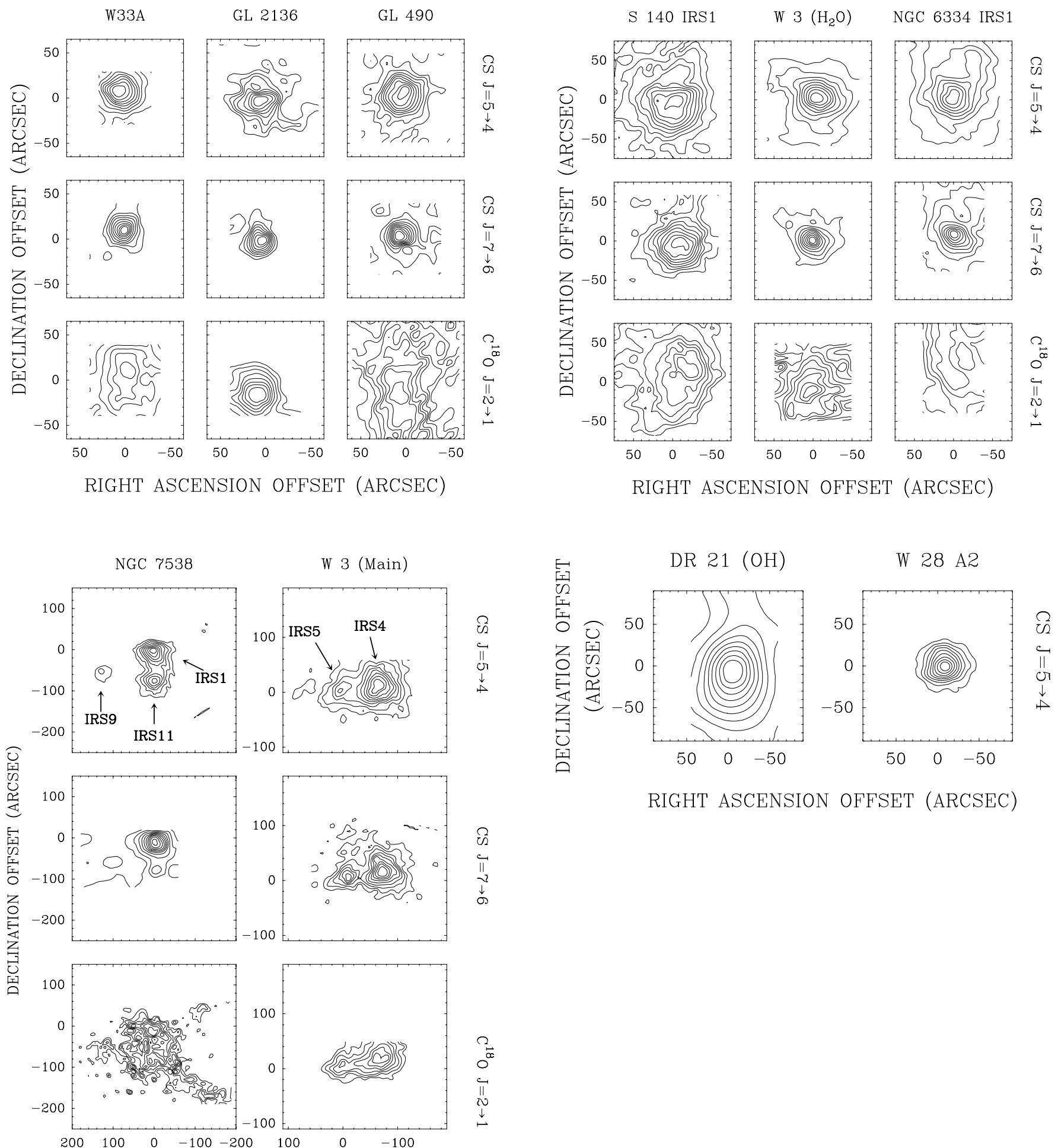

RIGHT ASCENSION OFFSET (ARCSEC)

RIGHT ASCENSION OFFSET (ARCSEC)

FIG. 3.-CSO maps of the $\mathrm{C}^{18} \mathrm{O} J=2 \rightarrow 1$ and CS $J=5 \rightarrow 4$ and $7 \rightarrow 6$ lines. Contours are $10 \%$ of the peak flux, starting at $10 \%$ for CS and $30 \%$ for $\mathrm{C}^{18} \mathrm{O}$. Integration intervals and peak $\mathrm{C}^{18} \mathrm{O}$ fluxes are: $19-26 \mathrm{~km} \mathrm{~s}^{-1}$ and $3.5 \mathrm{~K} \mathrm{~km} \mathrm{~s}^{-1}$ for GL $2136,-15$ to $-10 \mathrm{~km} \mathrm{~s}^{-1}$ and $4.0 \mathrm{~K} \mathrm{~km} \mathrm{~s} \mathrm{kor} \mathrm{GL}^{-1}$, -18 to $0 \mathrm{~km} \mathrm{~s}^{-1}$ and $7.7 \mathrm{~K} \mathrm{~km} \mathrm{~s}^{-1}$ for NGC $6334,-65$ to $-50 \mathrm{~km} \mathrm{~s}^{-1}$ and $5.0 \mathrm{~K} \mathrm{~km} \mathrm{~s}^{-1}$ for NGC 7538, -12 to $-2 \mathrm{~km} \mathrm{~s}^{-1}$ and $3.5 \mathrm{~K} \mathrm{~km} \mathrm{~s}$ for S140, $30-44$ $\mathrm{km} \mathrm{s}^{-1}$ and $3.8 \mathrm{~K} \mathrm{~km} \mathrm{~s}^{-1}$ for W33A, -58 to $-38 \mathrm{~km} \mathrm{~s}^{-1}$ and $5.1 \mathrm{~K} \mathrm{~km} \mathrm{~s}^{-1}$ for $\mathrm{W} 3\left(\mathrm{H}_{2} \mathrm{O}\right)$, and -50 to $-32 \mathrm{~km} \mathrm{~s}^{-1}$ and $5.2 \mathrm{~K} \mathrm{~km} \mathrm{~s}^{-1}$ for W3 (Main). See Table 2 for the peak fluxes of the CS lines.

binary star at a separation of 4.7 or 19,000 AU. This separation is larger than the $\sim 10^{3}$ AU found by Wyrowski et al. (1999) for W3 $\left(\mathrm{H}_{2} \mathrm{O}\right)$ and by Mundy, Looney, \& Welch (2000) for a sample of low-mass YSOs, but comparable to that found by Woody et al. (1989) and Padin et al. (1989) for DR $21(\mathrm{OH})$.

The flux densities found here for NGC 7538 IRS 1 are in agreement with those by Woody et al. (1989) and Akabane 

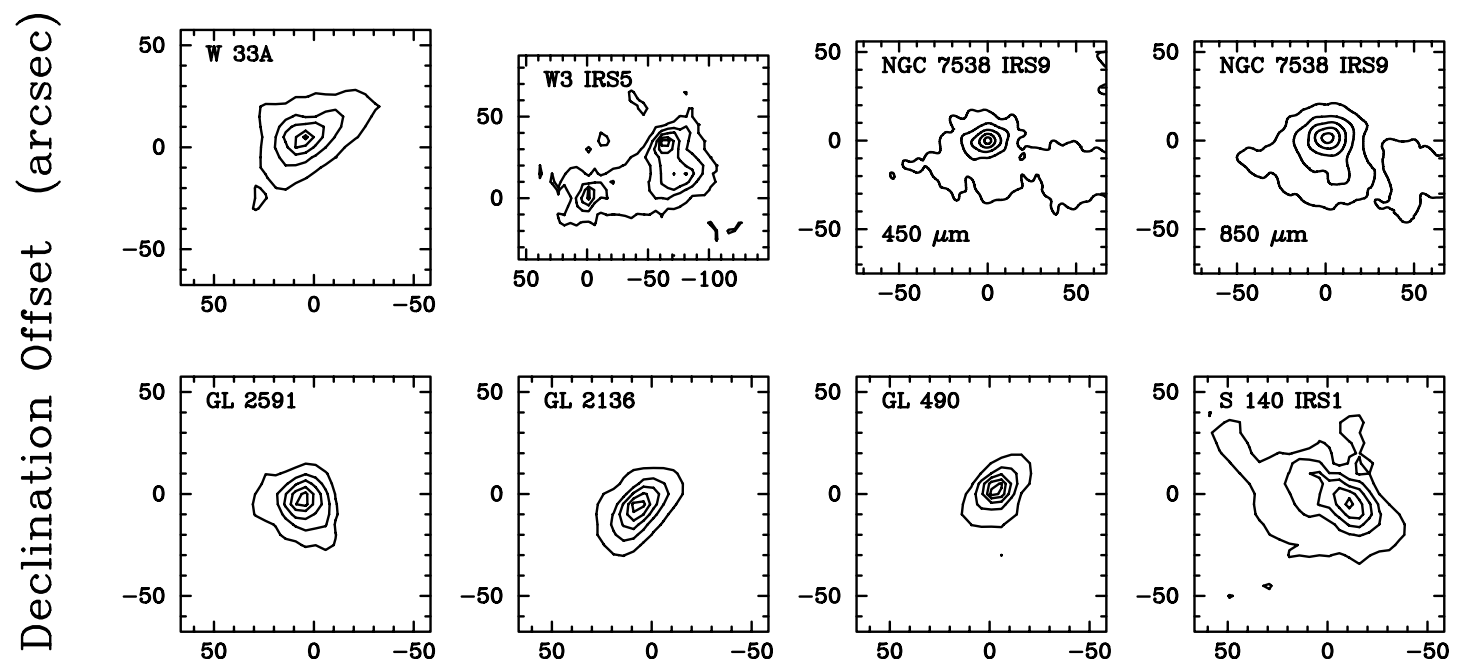

\section{Right Ascension Offset (arcsec)}

FIG. 4.-Maps of $350 \mu \mathrm{m}$ continuum emission made with SHARC, and of NGC 7538 at 450 and $850 \mu \mathrm{m}$, observed with SCUBA on the JCMT. Contours are $10 \%-90 \%$ of the peak brightness, in steps of $10 \%$. Peak brightness (in Jy beam ${ }^{-1}$ ) is 45.8 for W33A, 7.7 for W3 IRS $5,27.7$ for NGC 7538 IRS 9 at $450 \mu$ m and 4.8 at $850 \mu \mathrm{m}, 28.5$ for GL 2591, 34.5 for GL 2136, 13.4 for GL 490, and 37.7 for S140 IRS 1.

et al. (1992). The spectral index, measured from 107 to 230 $\mathrm{GHz}$, is $(0.9 \pm 0.5)$. The spectral indices of the sources in W33A are (1.8 \pm 0.6$)(\mathrm{MM} 1)$ and $(1.6 \pm 0.6)(\mathrm{MM} 2)$. The values of the spectral indices for W33A rule out optically thin emission from either ionized gas or from dust. They are, however, consistent with blackbody emission, probably from a compact dust source. In van der Tak et al. (1999),
OVRO observations of GL 2591 were presented which gave similar results; we refer the reader to that paper for a detailed discussion.

\section{MODELS}

Motivated by the results of the excitation analysis (§ 4.2) and of the CS maps ( $\$ 4.3$ ), we will proceed by developing
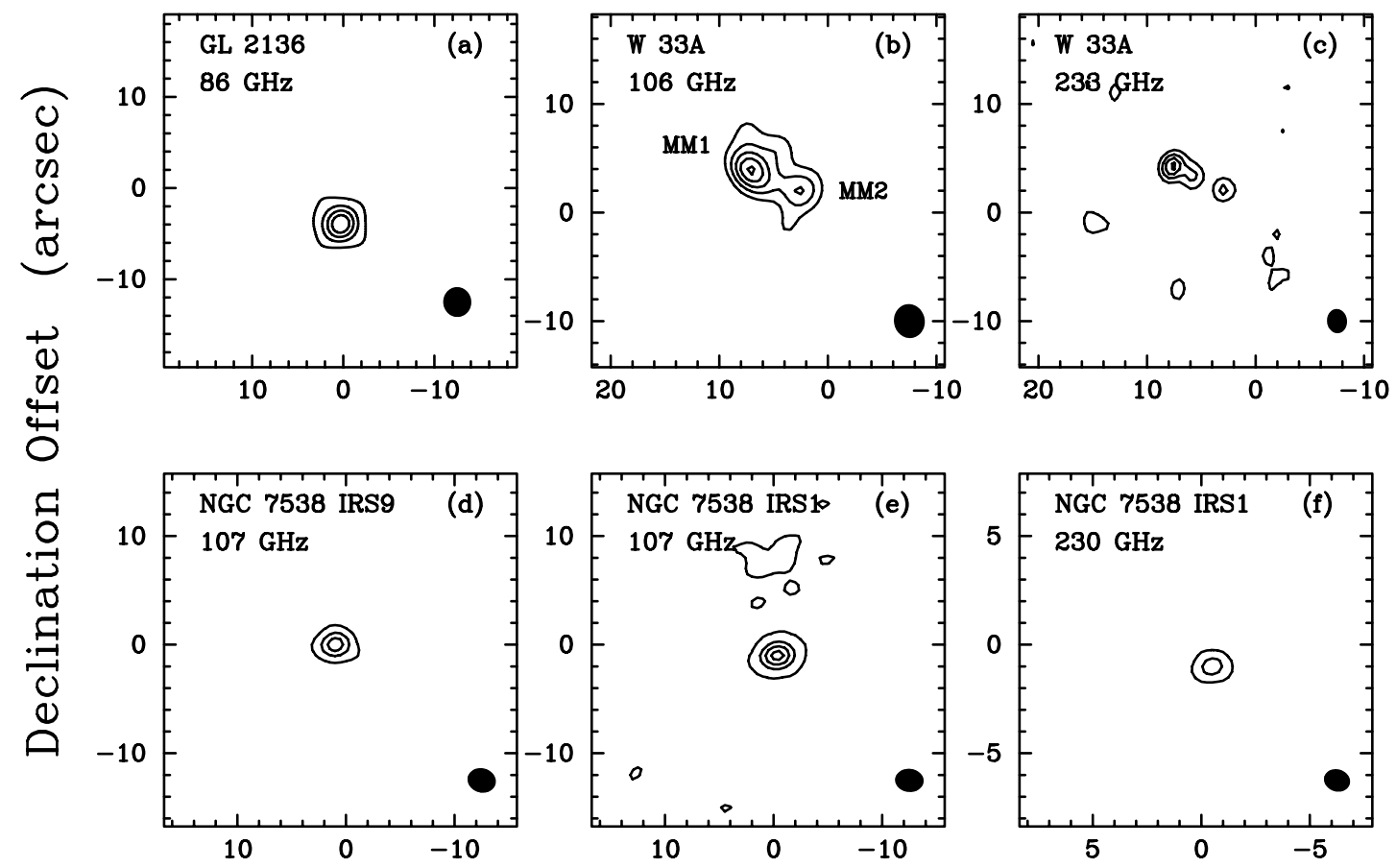

\section{Right Ascension Offset (arcsec)}

FIG. 5.-OVRO continuum maps of GL 2136, W33A, NGC 7538 IRS 1 and NGC 7538 IRS 9. Contour levels (in mJy beam $\left.{ }^{-1}\right)$ are: (a) 6, 18, 30, 42; (b) 8, $16,24,32,40,48,56 ;(c) 40,80,120,160,200,240,280 ;(d) 6,18,30,42 ;(e) 50,550,1050,1550 ;(f) 500,2000,3500,5000,6500,8000$. Beam sizes and shapes are drawn in the bottom right-hand corner of each panel. 
spherically symmetric power-law models. The modeling procedure follows van der Tak et al. (1999), to which we refer for details.

\subsection{Dust Continuum Models: Mass and Temperature Structure}

The dust emission from the sources was modeled using the one-dimensional diffusion code by Egan, Leung, \& Spagna (1988). A density structure of the form $n=$ $n_{0}\left(r / r_{0}\right)^{-\alpha}$ was used, with $\alpha$ in the range $0.5-2.0$. The fiducial radius $r_{0}$ was set to be half the diameter of the CS emission $[D(C S)$ in Table 3] and converted to AU in Table 5. The density $n_{0}$ at this radius was derived for each $\alpha$ by matching the submillimeter dust emission; in the next section, we will constrain the value of $\alpha$ by modeling molecular lines. To avoid edge effects in modeling the full range of emission, we used outer radii in our models that are twice $r_{0}$. The inner radius was arbitrarily set to $1 / 300$ of the outer radius, small enough not to influence the calculated brightness or temperature profile, as verified by test calculations for W33A and GL 2591. Dust opacities appropriate for star-forming regions were taken from Ossenkopf \& Henning (1994) (their Model 5) and converted to absorption cross-sections using a grain mass density of $2.5 \mathrm{~g} \mathrm{~cm}^{-3}$ and a radius of $0.1 \mu \mathrm{m}$. This radius is close to the median of more realistic size distributions so that the calculated dust temperatures represent the bulk of the dust (cf. Churchwell, Wolfire, \& Wood 1990). Dust properties are known to vary from one region to the next (Lis et al. 1998; Visser et al. 1998; Hogerheijde \& Sandell 2000) and are likely to change inside our sources as well because of the changing temperature (Mennella et al. 1998; see $\S 6.2$ ). Maps at several far-infrared wavelengths at $\lesssim 10^{\prime \prime}$ resolution would allow us to disentangle temperature and dust opacity variations, but awaiting such data, we assume a constant grain opacity. With the luminosity, the distance, and the size of the source fixed, $M\left(<r_{0}\right)$ was used as the only free parameter to match the observed submillimeter continuum emission.

Figure 6 shows the synthetic continuum spectra compared with observations. Most submillimeter data are from this work and from Sandell (1994); the sources of the additional data are listed in the legend. The models have been constructed to fit the submillimeter $(300-1300 \mu \mathrm{m})$ data and are seen to reproduce the observed emission at $\gtrsim 50 \mu \mathrm{m}$ for every source, i.e., up to optical depths of a few. The shorter wavelength emission is in general not matched, although care was taken to include only photometry in small $\left(\lesssim 10^{\prime \prime}\right)$ beams to avoid a contribution from reflection nebulosities. The high brightness of GL 2591 at $20 \mu \mathrm{m}$ was attributed by van der Tak et al. (1999) to the evaporation of ice mantles close to the star, which decreases the $20 \mu \mathrm{m}$ optical depth by $30 \%$ (Ossenkopf \& Henning 1994). Similar effects play a role for the sources presented here, as illustrated for W33A in the figure. The emission at $\lambda \lesssim 10 \mu \mathrm{m}$ is not expected to be well reproduced in these models, since the high optical depth makes it very sensitive to deviations from the assumed spherical shape. That all sources require less extinction at short wavelengths is discussed quantitatively in $\S 6.1$. This point is illustrated by the fact that the column densities derived in this paper are much higher than those found by Faison et al. (1998) by fitting the near- to farinfrared spectra of a sample of ultracompact $\mathrm{H}$ II regions, which have similar submillimeter flux densities and lie at similar distances.

The calculated temperatures follow an $r^{-0.4}$ profile at distances greater than $\sim 2-3 \times 10^{3}$ AU from the star, with the absolute temperature scale set by the luminosity. Inside this radius, the envelope is optically thick to the photons carrying most of the energy, and the temperature gradient is steeper than $r^{-0.4}$, with the absolute scale set by the extinction, which acts as a blanket. Hence, for a given luminosity, a lower extinction leads to a higher temperature at a certain radius. This is the case for our models with shallow density gradients. For a given column density, a shallower density gradient implies a lower extinction (in a pencil beam) because a larger fraction of the beam is filled with warm dust. We therefore expect, like Chini et al. (1986) and Churchwell et al. (1990), that bright mid-infrared sources have shallow density gradients. However, Figure 6 shows that even the models with $\alpha=0.5$ fail to fit the near-infrared

TABLE 5

Parameters of Best-Fit Models

\begin{tabular}{|c|c|c|c|c|c|c|c|c|c|}
\hline $\begin{array}{l}\text { Source } \\
\text { (1) }\end{array}$ & $\begin{array}{c}r_{0} \\
\left(10^{3} \mathrm{AU}\right) \\
(2)\end{array}$ & $\begin{array}{c}\alpha \\
(3)\end{array}$ & $\begin{array}{c}n_{0} \\
\left(10^{4} \mathrm{~cm}^{-3}\right) \\
(4)\end{array}$ & $\begin{array}{c}M\left(<r_{0}\right) \\
\left(M_{\odot}\right) \\
(5)\end{array}$ & $\begin{array}{c}M_{V} \\
\left(M_{\odot}\right) \\
(6)\end{array}$ & $\begin{array}{c}\bar{T} \\
(\mathrm{~K}) \\
(7)\end{array}$ & $\begin{array}{c}\mathrm{CO} / \mathrm{H}_{2} \\
\left(\times 10^{4}\right) \\
(8)\end{array}$ & $\begin{array}{c}\mathrm{CS} / \mathrm{H}_{2} \\
\left(\times 10^{9}\right) \\
(9)\end{array}$ & $\begin{array}{c}\mathrm{H}_{2} \mathrm{CO} / \mathrm{H}_{2} \\
\left(\times 10^{9}\right) \\
(10)\end{array}$ \\
\hline W3 IRS $5 \ldots \ldots \ldots \ldots$ & 60 & 1.5 & 2.3 & 262 & 355 & 33 & 1.6 & 5. & 3. \\
\hline GL $490 \ldots \ldots \ldots \ldots \ldots$ & 19 & 1.5 & 8.6 & 30 & 168 & 19 & 0.4 & 1. & 1. \\
\hline W33A................. & 74 & 1.0 & 6.8 & 1089 & 1984 & 20 & 0.5 & 5. & 4. \\
\hline GL $2136 \ldots \ldots \ldots \ldots \ldots$ & 35 & 1.25 & 3.6 & 72 & 316 & 28 & 1.2 & 4. & 8. \\
\hline GL 7009S ............. & 91 & 0.5 & 17. & 3955 & 2218 & 21 & 0.3 & $0.4-0.8$ & $\ldots$ \\
\hline GL $2591 \ldots \ldots \ldots \ldots \ldots$ & 27 & 1.0 & 5.8 & 44 & 268 & 28 & 1.5 & 10. & 4. \\
\hline S140 IRS $1 \ldots \ldots \ldots \ldots$ & 29 & 1.5 & 3.6 & 46 & 128 & 27 & 1.0 & 5. & 5. \\
\hline NGC 7538 IRS $1 \ldots \ldots$ & 72 & 1.0 & 5.3 & 815 & 1128 & 25 & 0.6 & 10. & 10. \\
\hline NGC 7538 IRS $9 \ldots \ldots$ & 66 & 1.0 & 3.9 & 430 & 1016 & 20 & 0.3 & 10. & 10. \\
\hline IRAS $20126 \ldots \ldots \ldots \ldots$ & 39 & 1.75 & 2.2 & 84 & 294 & 27 & 0.5 & 3. & $\ldots$ \\
\hline DR $21(\mathrm{OH}) \ldots \ldots \ldots .$. & 29 & 1.75 & 23. & 350 & 429 & 15 & 0.8 & 3. & 5. \\
\hline W3 $\left(\mathrm{H}_{2} \mathrm{O}\right) \ldots \ldots \ldots \ldots \ldots$ & 45 & 2.0 & 5.3 & 385 & 932 & 26 & 1.0 & 10. & 3. \\
\hline NGC 6334 IRS $1 \ldots \ldots$ & 43 & 2.0 & 6.2 & 382 & 725 & 35 & 1.5 & 12. & 7. \\
\hline W28 A2 ............... & 37 & 1.5 & 9.9 & 292 & 982 & 42 & 1.7 & 20. & $\ldots$ \\
\hline
\end{tabular}

NoTe-The parameters in columns (2)-(4) specify the density structure in the form $n(r)=n_{0}\left(r / r_{0}\right)^{-\alpha}$, with the reference radius $r_{0}$ half the diameter listed in Table 3. Column (5) gives the integrated mass enclosed within $r_{0}$, and column (6) the virial mass within $r_{0}$. Column (7) gives the mass-weighted temperature in the models; columns (8)-(10) provide the derived abundances of CO, CS, and $\mathrm{H}_{2} \mathrm{CO}$. 


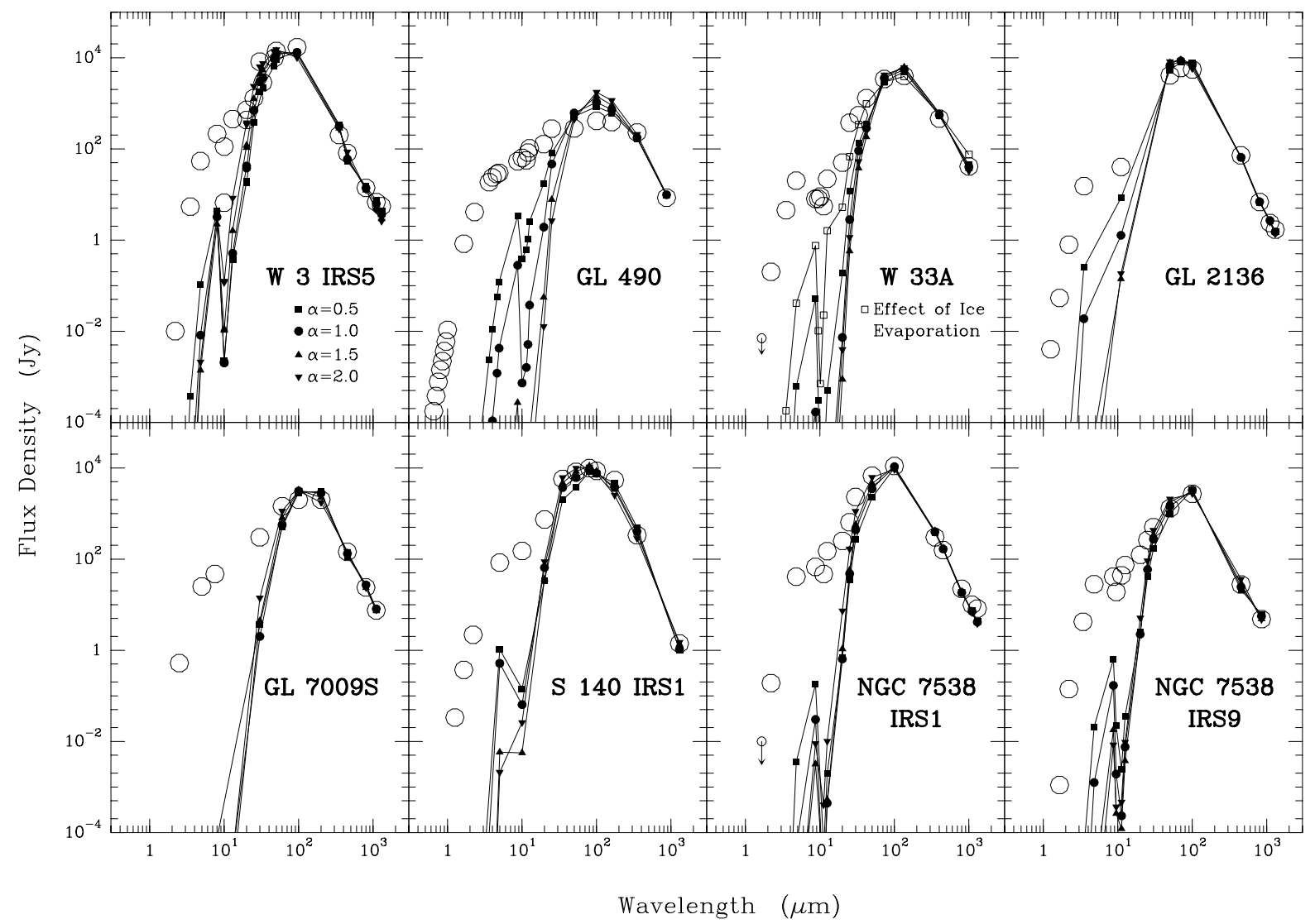

FIG. 6.-Observed continuum spectra (open circles) and models for several values of $\alpha$. The symbol legend is in the top left-hand panel of $6 a$. For W33A, a model using grains without ice mantles is also shown. Data are taken from Campbell et al. 1995 for W3 IRS 5; Chini et al. 1991 for GL 490; Moorwood \& Salinari 1981, Evans et al. 1979, Dyck \& Simon 1977, Stier et al. 1984, Jaffe et al. 1984, and Cheung et al. 1980 for W33A; Allen et al. 1977, Lebofsky et al. 1976, E. F. van Dishoeck (1998, private communication), and Kastner et al. 1994 for GL 2136; Dartois et al. 1998a and McCutcheon et al. 1995 for GL 7009S; Evans et al. 1989, Willner et al. 1982, and Zhou et al. 1994 for S140 IRS 1, Werner et al. 1979 for NGC 7538 IRS 1; Werner et al. 1979 and G. Sandell (1998, private communication) for NGC 7538 IRS 9; Wilking et al. 1990 and Cesaroni et al. 1997, 1999 for IRAS 20126 + 4104; Thum \& Lemke 1975, Harvey et al. 1986, Harvey et al. 1977, Richardson et al. 1989, and Chandler et al. 1993 for DR 21(OH); Wynn-Williams et al. 1972, Keto et al. 1992, and Thronson \& Harper 1979 for W3 $\left(\mathrm{H}_{2} \mathrm{O}\right)$; Straw \& Hyland 1989 and Harvey \& Gatley 1983 for NGC 6334 IRS 1; and Moorwood \& Salinari 1981, Lightfoot et al. 1984, and Harvey et al. 1994 for W28 A2.

part of the spectrum. This is not because of our selection on brightness in the mid-infrared since the data of the comparison samples are not matched either. The reason for the discrepancy must be sought in (small) geometrical effects.

\subsection{Models of the Line Emission: Density Structure}

To determine the slope of the density gradient $\alpha$, the $\mathrm{C}^{17} \mathrm{O}, \mathrm{CS}, \mathrm{C}^{34} \mathrm{~S}$, and $\mathrm{H}_{2} \mathrm{CO}$ line radiation from the 14 sources was modeled with the Monte Carlo code written by M. R. Hogerheijde \& F. F. S. van der Tak $(2000$, in preparation). The models consist of 30 spherical shells spaced logarithmically within the same radii as the dust models. The same density structures were used as in the dust models, and the temperature structure was taken from the dust continuum models for the same value of $\alpha$. The intrinsic line profile was assumed to be a Gaussian with the measured FWHM (Table 3). Initially, molecular abundances were assumed to be constant throughout the model.

The gas column density [or equivalently $M\left(<r_{0}\right)$ or the value of $n_{0}$ ] for each $\alpha$ was also taken from the dust models, assuming a gas to dust mass ratio of 100 . This assumption was tested for GL 2591 against $\mathrm{C}^{17} \mathrm{O}$ observations by van der Tak et al. (1999) because this source has negligible depletion of $\mathrm{CO}$ (see also $\S 6.2$ ). With the column density fixed, the density profile can be obtained by modeling the emission lines of the high critical density molecules CS and $\mathrm{H}_{2} \mathrm{CO}$. After solving for the molecular excitation, velocityintegrated intensities have been calculated in the appropriate beam for each observation. In many cases, a line was observed in several beams, which in our models acts as a simple substitute for simulating map data. Comparison to the observations proceeds by minimizing the quantity

$$
\chi^{2} \equiv \frac{1}{N} \Sigma\left[\frac{F^{(\text {obs })}-F^{(\text {model })}}{\sigma}\right]^{2}
$$

where $F^{(\text {obs })}$ and $F^{(\text {model })}$ are the observed and synthetic line fluxes and the sum is taken over all $N$ observed lines. A grid of models was run for each molecule by varying the densitylaw exponent $\alpha$ and the molecular abundance.

The success of any fit procedure depends critically on a good understanding of the error budget $(\sigma)$. Most line fluxes were assigned a $30 \%$ error, but the following lines have a larger uncertainty of $50 \%$ : (1) lines in the $460-490 \mathrm{GHz}$ band, because of the more uncertain calibration; (2) lines measured in beams of $\gtrsim 30^{\prime \prime}$ diameter, which may suffer from confusion; and (3) the $J=1 \rightarrow 0$ lines of $C S$ and $C^{34} S$, which may contain a contribution from the surrounding cloud. The following observations were found to be more than a factor of 2 off any of our models and were therefore discarded in the $\chi^{2}$ calculation: CS $J=5 \rightarrow 4$ and $7 \rightarrow 6$ in 


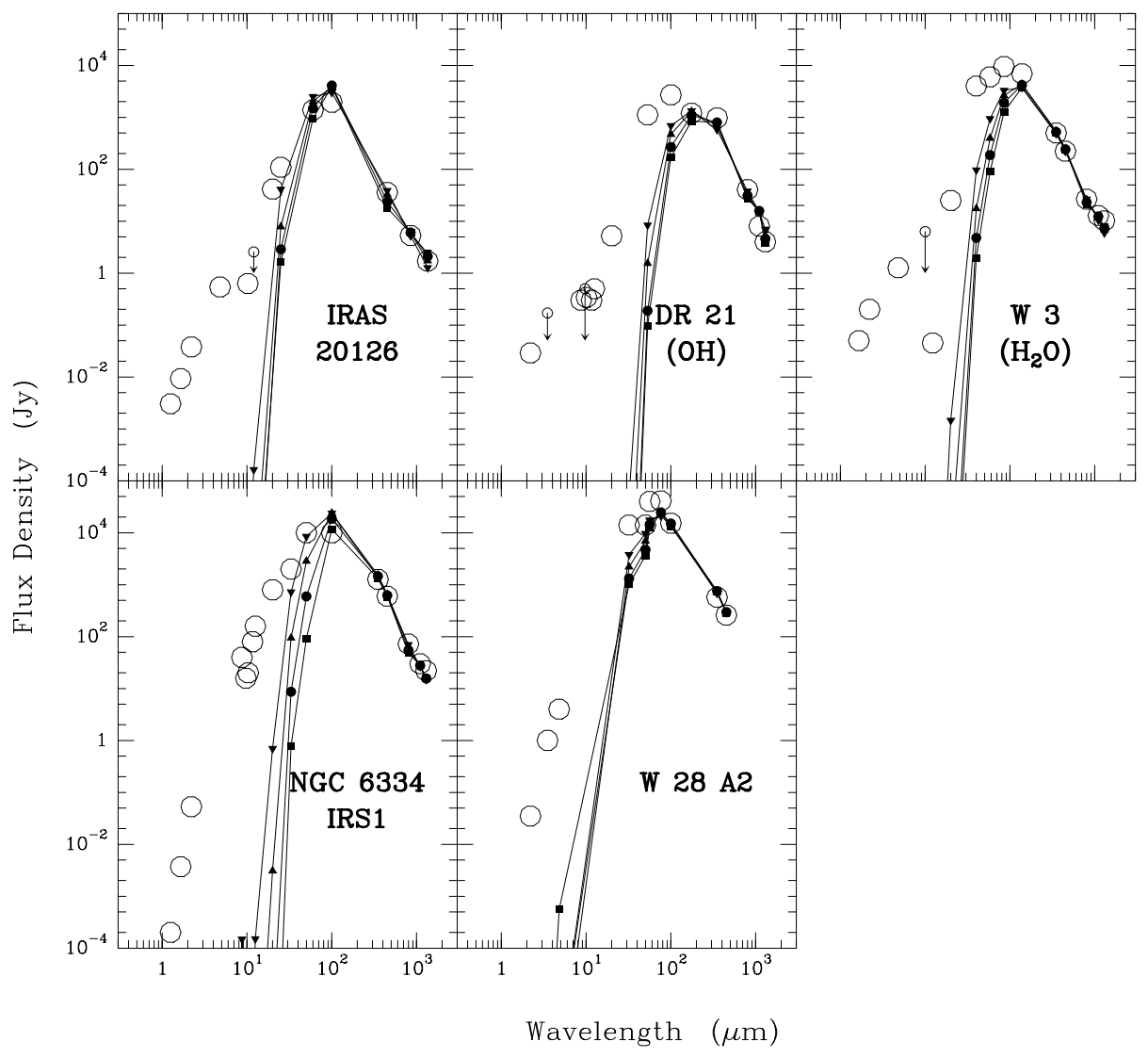

Fig. 6.-Continued

GL 490 and CS $7 \rightarrow 6$ in W33A from Plume et al. (1997), CS $5 \rightarrow 4$ and $C^{34}$ S $3 \rightarrow 2$ in S140 IRS 1 from Zhou et al. (1994), $\mathrm{C}^{34} \mathrm{~S} 2 \rightarrow 1$ in IRAS $20126+4104$ from Cesaroni et al. (1997) and $C^{34} S J=7 \rightarrow 6$ in W28 A2 from Plume et al. (1997).

The results of the emission-line models are presented in Figure 7. The fit quality parameter $\chi^{2}$ is plotted as a function of the density-law exponent $\alpha$ and the CS abundance with respect to $\mathrm{H}_{2}$. The elongation of the $\chi^{2}$ contours indicates that the quality of the fit depends to first order on the CS abundance and to second order on the density profile. The parameters of the best-fitting models are summarized in Table 5.

Table 5 shows that, for the main sample, $\alpha=1.0-1.5$, while the other sources have $\alpha=1.75-2.0$. This result is consistent with the result from the dust models ( $\S 5.1$ ), namely, that for a given source, the model with lowest $\alpha$ has the highest mid-infrared flux. Thus, the mid-infrared brightness of the main sample is not a pure orientation effect. However, since the spherical dust models fail to reproduce the near-infrared emission of all sources, deviations from spherical symmetry are important. This conclusion is supported by synthetic CS line profiles from our power-law models, which are self-absorbed for all but the least massive sources. The effect of deviations from spherical symmetry on line profiles and near-infrared emission was discussed for GL 2591 in van der Tak et al. (1999). Decreasing the central column density by a factor of a few can increase the midinfrared emission and decrease the self-absorption substantially.

The best-fit CS abundance is $3 \times 10^{-9}$ on average, with source-to-source variations of a factor of 3 for most sources.
The hot cores and the ultracompact $\mathrm{H}$ II region have the highest CS abundances: $(1-2) \times 10^{-8}$. The low CS abundance in GL 7009S, $0.4 \times 10^{-9}$, is probably caused by freezing out of the molecules onto the dust grains, as suggested by the large column densities of several ice species toward this source (d'Hendecourt et al. 1996).

Figure 7 also lists the number of $\mathrm{CS}$ and $\mathrm{C}^{34} \mathrm{~S}$ lines observed. The sources for which the most lines have been observed also have the tightest constraints on $\alpha$. For less well-observed sources, our results may be influenced by sampling effects. In particular, if only a small range of molecular energy levels is available, the value of $\alpha$ may be biased, or a gradient may be hard to detect. We have checked for such effects by calculating $\bar{J}$, the average upper $J$-level of the data set. Most sources have $\bar{J}=4.5-5.5$, which implies that the observable range of critical densities has been evenly sampled. However, for GL 7009S, and IRAS 20126, for which fewer high-excitation line data exist, $\bar{J}=$ $3.2-3.6$, and the model results are therefore less robust.

The value $\alpha=2$ found for the hot cores is higher than that found for the other sources, and we have investigated if this result could be an artifact of our assumption of a constant molecular abundance. Hot cores have high abundances of many molecules, including saturated organics thought to be caused by freshly evaporated grain mantles. In this picture, the radius where the temperature reaches $90 \mathrm{~K}$ is an important threshold because water ice, the main ingredient of interstellar grain mantles, evaporates. It is possible that the abundance of CS is enhanced above this temperature as well. To test this idea, a model has been run with the luminosity and the $M\left(<r_{0}\right)$ of $\mathrm{W} 3\left(\mathrm{H}_{2} \mathrm{O}\right)$, but with $\alpha=1.5$. The CS abundance is $5 \times 10^{-9}$ in the outer parts 


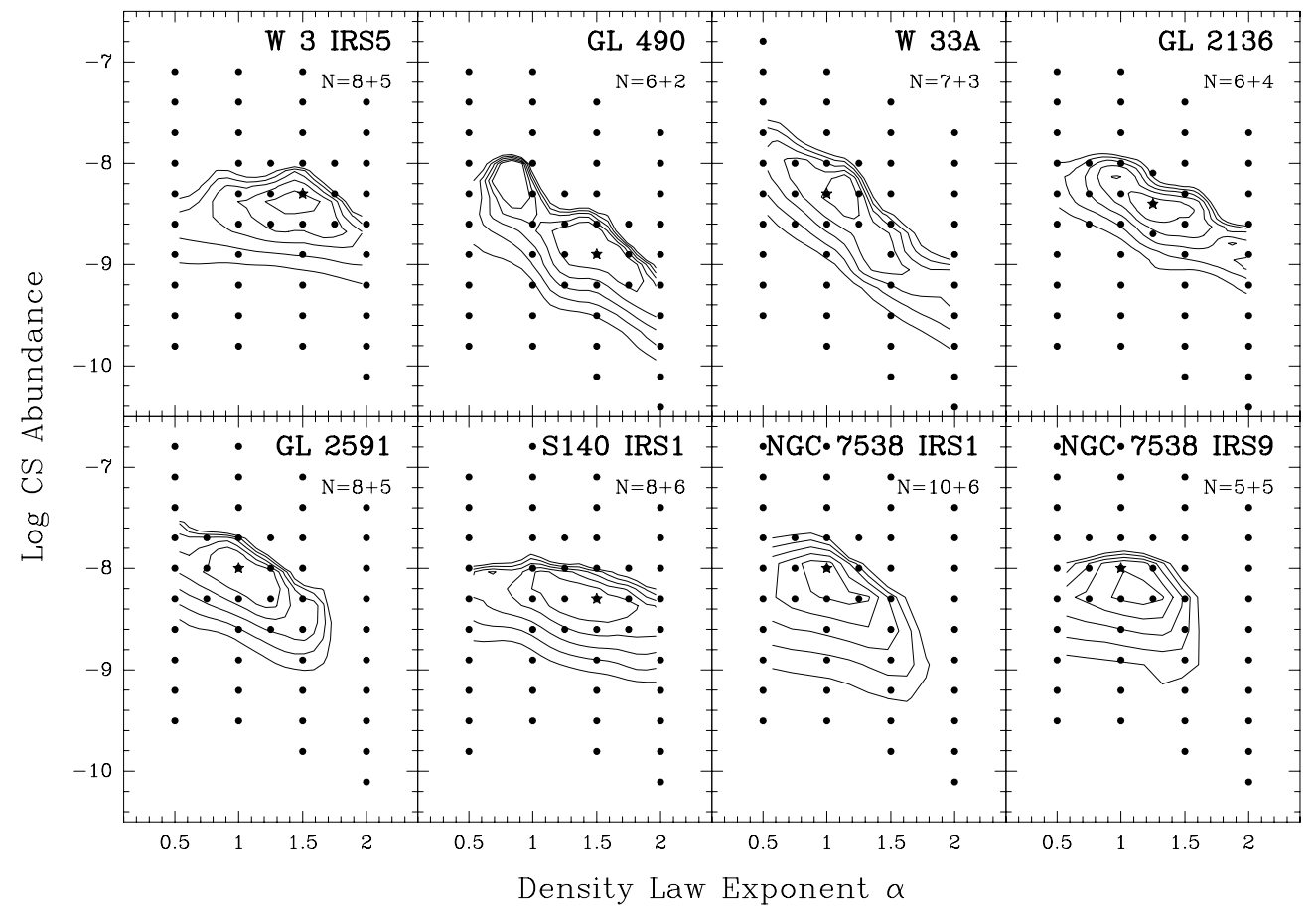

FIG. 7.-Fit quality parameter $\chi^{2}$ plotted as a function of density-law exponent $\alpha$ and CS abundance. Contours increase by 1 and start at 1 for W3 IRS 5 , GL 7009S, IRAS 20126, W3 $\left(\mathrm{H}_{2} \mathrm{O}\right)$, and NGC 6334 IRS 1; 3 for NGC 7538 IRS 1 and NGC 7538 IRS 9; and at 2 for the other seven sources. The best-fitting model is indicated by a star. In the top right-hand corner, the number of CS and $\mathrm{C}^{34} \mathrm{~S}$ lines included in the $\chi^{2}$ calculation is listed.

but enhanced where the dust temperature exceeds $90 \mathrm{~K}$. A match to the data of equal quality as with the best constantabundance model $\left(\alpha=2.0, \mathrm{CS} / \mathrm{H}_{2}=1 \times 10^{-8}\right.$ : Table 5) was obtained by enhancing the CS abundance in the inner region by a factor of 10 . This result indicates that the hot cores may have a density structure similar to that of the main sample, if plausible variations of $\mathrm{CS} / \mathrm{H}_{2}$ with radius are considered. SCUBA maps of other hot-core-type sources by Hatchell et al. (2000) indeed suggest values of $\alpha \approx 1.5$.

\subsection{Comparison of Dust and Gas Tracers}

The density structures derived from the CS excitation will now be tested by using them to model the radial profiles of the submillimeter dust emission. This emission is optically thin and, hence, measures the column density distribution, given the calculated temperature structure. The points in Figure 8 are averages of slices along the north-south and east-west directions through the images shown in Figure 4. To reduce the noise, the data have been folded about the image maximum. In the case of W3 IRS 5, the local maximum in the SHARC map that corresponds to the infrared position was used to center the slices, and the western direction was not included in the scans because of confusing extended emission clearly visible in Figure 4. Superposed are slices through model images for various values of $\alpha$ as dotted lines, while the solid line corresponds to the value of $\alpha$ derived in $\S 5.2$ from the CS excitation. The model profiles (in a $10^{\prime \prime}$ beam) were calculated with a code kindly supplied by L. Mundy. The models are exactly those used to produce Figure 7, except that for W3 IRS 5, GL 490, and NGC 7538 IRS 9, the outer radii were increased to $60^{\prime \prime}$ to avoid edge effects. In these cases, the radial profile of the dust emission was quite extended.

Figure 8 demonstrates that the dust and gas tracers agree very well on the best value of $\alpha$, implying that the volume density and column density distributions are consistent. This result indicates that the dust and gas are well mixed and that the structure of the envelopes is fairly homogeneous and not very clumpy. The only exception to this rule is S140 IRS 1, for which the dust gives $\alpha=1.0$ while the gas gives $\alpha=1.5$. Although an inhomogeneous (clumpy) structure would cause a discrepancy in this direction, it is hard to see why only one of our sources would have such a structure. Clumps in S140 have been proposed by, e.g., Spaans \& van Dishoeck (1997), but in a much larger ( arcmin-sized) region outside the dense star-forming core. More likely to be important here is an elevated temperature caused by the nearby sources IRS 2 and IRS 3 at $10^{\prime \prime}-15^{\prime \prime}$ offsets, which have luminosities similar to that of IRS 1 (Evans et al. 1989), and by external heating by the nearby ionization front (Lester et al. 1986). So S140 is unique in our sample in that it is the only source where our assumption of one central heating source breaks down.

Although the data on GL 2591 are well matched by the power law on average, the slope of the data is shallower inside a radius of $10^{\prime \prime}-20^{\prime \prime}$ and drops more steeply outside this radius. This suggests a variation in the temperature or the density gradient with radius. Since the effect is more pronounced toward shorter wavelengths, a variation of the temperature gradient with radius seems more plausible. An inner region of roughly constant temperature, such as caused by a central cavity with little or no extinction, may reproduce the data, but such models fall outside the scope of this paper.

\section{DISCUSSION}

We have modeled single-dish continuum and line data with power-law models $n=n_{0}\left(r / r_{0}\right)^{-\alpha}$ and obtained good 


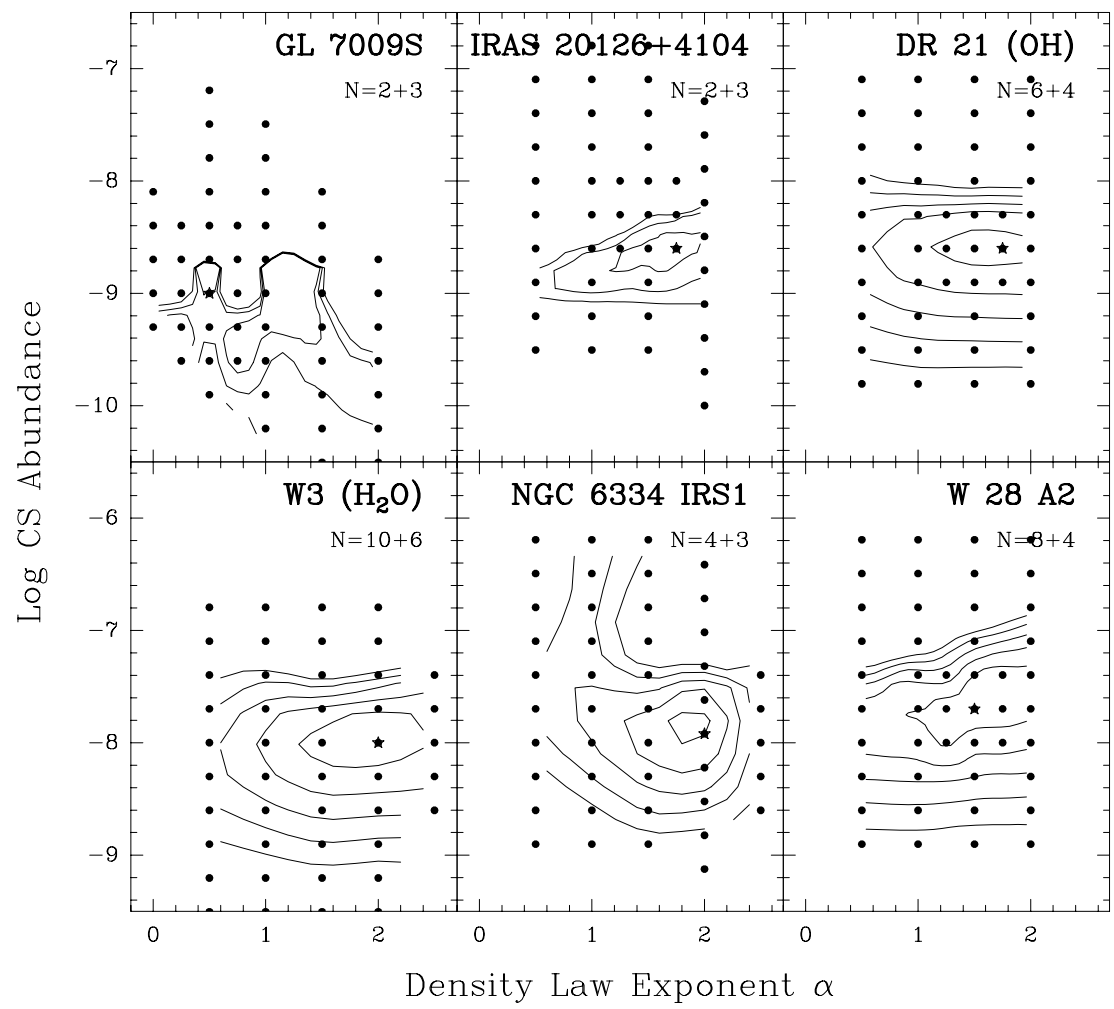

FIG. 7.-Continued

solutions $\left(\chi^{2}=1-3\right)$ with $\alpha \approx 1.0-1.5$ for the bright midinfrared sources and $\alpha=1.5-2.0$ for the other sources, although the highest value $\alpha=2.0$ found for two "hot cores" may be an overestimate caused by an enhanced CS abundance close to the center. This section investigates the validity of the assumptions that went into the models and discusses possible implications of their results.

\subsection{Envelope Structure}

The models developed in $\S 5$ assume that the clouds are spherical and homogeneous. Mitchell et al. (1990) observed the ${ }^{13} \mathrm{CO} v=1 \leftarrow 0$ band at $4.7 \mu \mathrm{m}$ in absorption toward the bright mid-infrared sources. The excitation of the quiescent (non-outflowing) gas rules out single-temperature models, but the data are well fitted by a model with two discrete temperatures. The column density ratio of these "cold" and "hot" components varies from $\approx 1$ to $\approx 5$. The physical origin of such a two-temperature structure is not clear, however. We have calculated the column densities in each rotational state of ${ }^{13} \mathrm{CO}$ up to $J=25$ from our powerlaw models, and the results are compared with the data of Mitchell et al. in Figure 9. The low-resolution data for GL 7009S from Dartois et al. (1998b) may contain a contribution from outflowing gas and, hence, are regarded as upper limits. The models have been scaled to the observed total column density, which is always within a factor of 2 from the column density measured by our submillimeter emission data (§ 4.2). The good match (maximum deviation of a

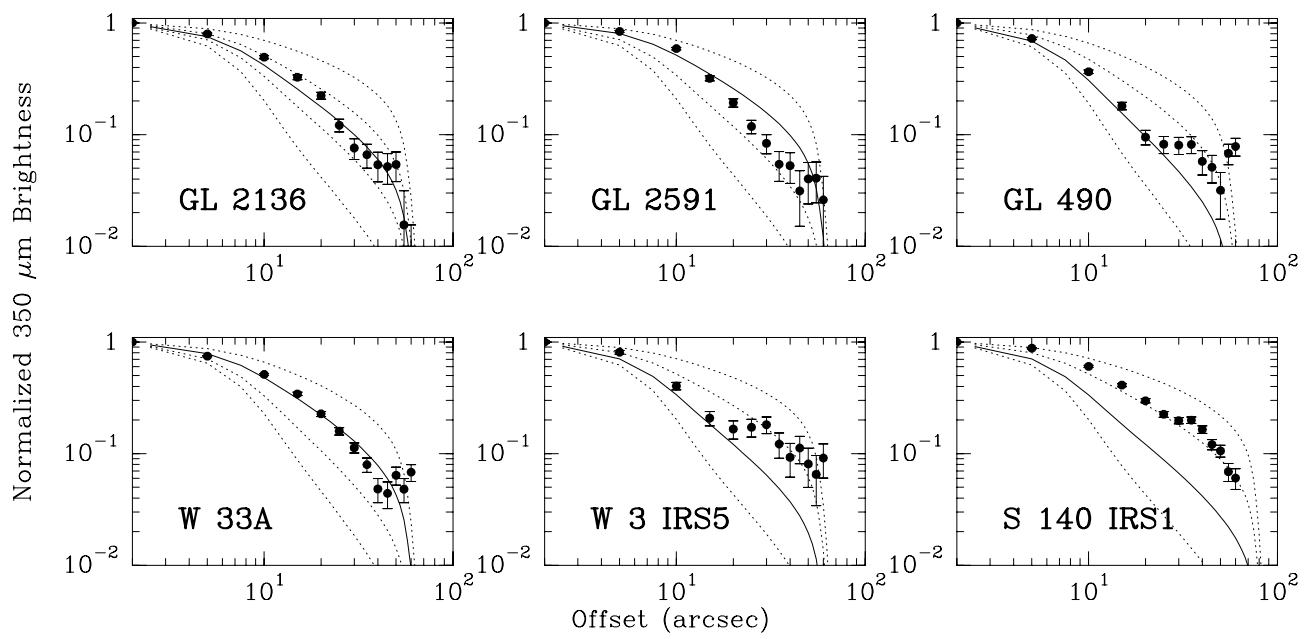

FIG. 8.-Radial profiles of $350 \mu \mathrm{m}$ emission observed with $(a)$ SHARC and $(b)$ SCUBA. Dotted lines are model fits from the power-law models for $\alpha=0.5,1.0,1.5$, and 2.0 (top to bottom). The solid line is the model that fits the CS excitation best. 


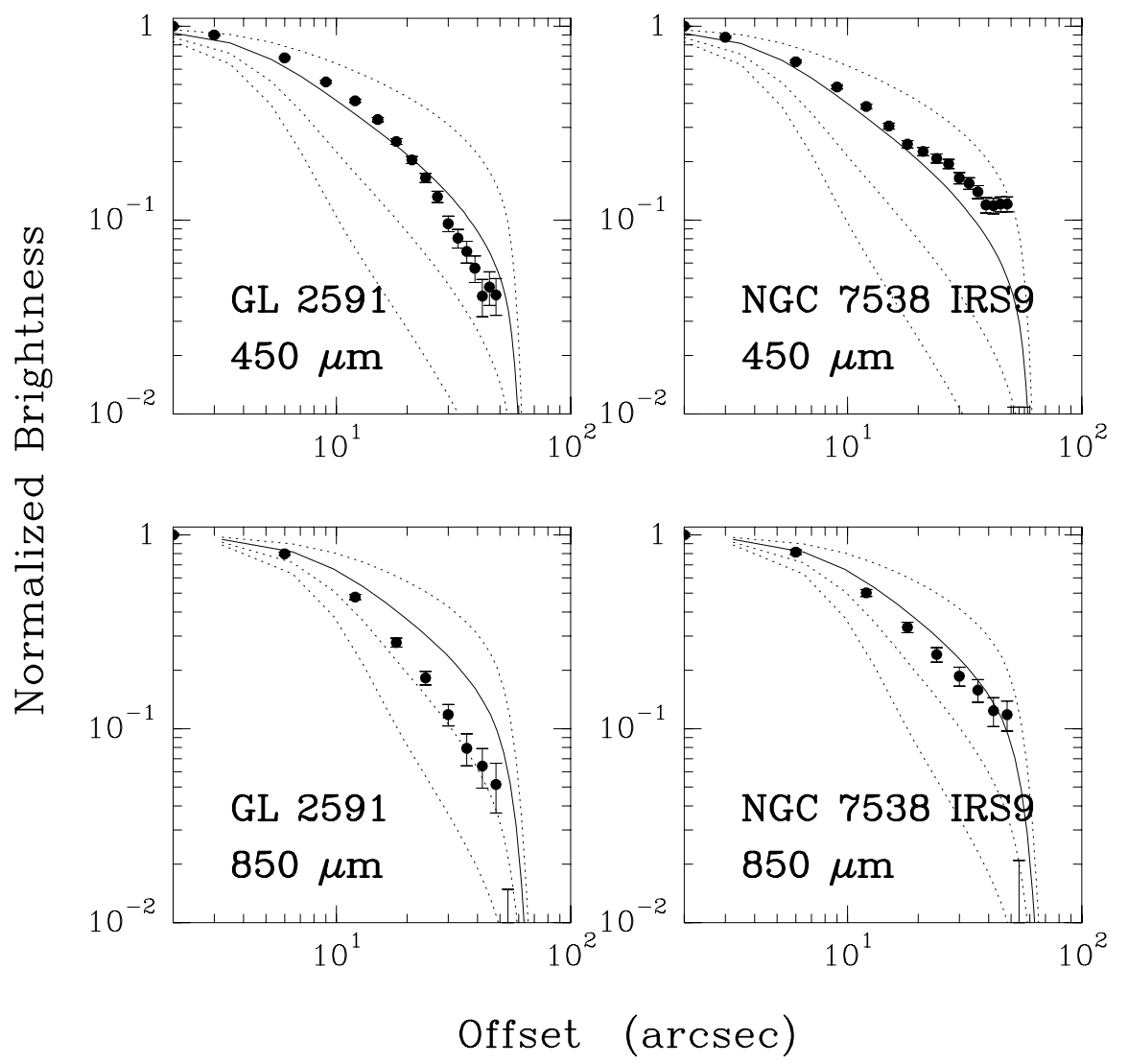

Fig. 8.-Continued

factor of 3) to the infrared data, which sample a pencil beam toward the infrared source, indicates that a spherically symmetric model is a reasonable first-order description of these sources. Together with the evidence from the CS emission line profiles and the near-infrared continuum emission, we estimate the deviations from spherical geometry as a decrease in optical depth by a factor of $\approx 3$ over an $\lesssim 10^{\prime \prime}$ area.

The assumption of a homogeneous density structure is tested in Figure 10, where $M\left(<r_{0}\right)$ is compared with the mass derived from the virial theorem inside the same radius. The latter method assumes that the cloud's gravitation just balances its pressure as measured by the line width $\Delta V$ (Table 3). If the gas is clumpy, the virial mass is smaller than the power-law mass by a fraction $f_{v}$, with the volume filling factor $0<f_{v}<1$. The ratio $M_{V} / M\left(<r_{0}\right)$ has a mean value of 2.77 and a standard deviation of 1.66. The only source for which $M\left(<r_{0}\right)>M_{V}$ is GL $7009 \mathrm{~S}$, for which the model results are uncertain.

While there is no evidence for clumping within the envelopes, other authors have found that regions of massive star formation are usually clumped on a much larger scale. It may not be a coincidence that the sizes derived for the clumps in S140 by Plume, Jaffe, \& Keene (1994) and in M17 SW by Wang et al. (1993), $0.2 \mathrm{pc}$, are similar to the sizes of the envelopes found here. It is, however, outside the scope of this paper to investigate a possible evolutionary connection between molecular cloud clumps and the envelopes of massive young stars.

\subsection{Relation of $\mathrm{CO}$ Abundance with Temperature}

Our assumed values of the gas to dust mass ratio and the dust opacity at submillimeter wavelengths were tested by modeling $\mathrm{C}^{17} \mathrm{O} J=3 \rightarrow 2$ and $2 \rightarrow 1$ observations. The best-fit values for the abundance of $\mathrm{CO}$ are listed in column 6 of Table 5 . The derived abundances are $10 \%-60 \%$ of the value of $2.7 \times 10^{-4}$ measured by Lacy et al. (1994) toward the warm cloud NGC 2024. Our CO/ $\mathrm{H}_{2}$ value for GL 2591 is in good agreement with the value of $3.1 \times 10^{-4}$ measured by C. Kulesa (1999, private communication), while in the case of GL 490, our value is a factor of 7 lower. The CO abundance measured in emission is expected to be lower than in absorption because the emission data are more sensitive to extended cold gas. When measured in infrared absorption, which samples warm material close to the star, the column density ratio of solid to gaseous $\mathrm{CO}$ never exceeds unity toward massive young stars (Mitchell et al. 1990; van Dishoeck 1998).

Although source-to-source variations in grain opacity cannot be ruled out, a more likely explanation of the spread in $\mathrm{CO}$ abundances is that different amounts of $\mathrm{CO}$ are frozen out on grain surfaces. This occurs at temperatures $\lesssim 20 \mathrm{~K}$ in the case of pure CO (Sandford \& Allamandola 1993) and up to $\sim 45 \mathrm{~K}$ for $\mathrm{CO}-\mathrm{H}_{2} \mathrm{O}$ mixtures. In both cases, a correlation of the abundance with temperature is expected. Figure 11 shows a plot of the $\mathrm{CO}$ abundance derived from $\mathrm{C}^{17} \mathrm{O}$ observations versus the mass-weighted temperature, defined as $\bar{T}=\int T(r) n(r) r^{3} d r / \int n(r) r^{3} d r$ (see Table 5). The two quantities are seen to be correlated, which we interpret as the effect of depletion of $\mathrm{CO}$ at low temperatures.

These results strongly suggest that depletion and thermal desorption are the processes controlling the abundance of $\mathrm{CO}$ in the gas phase after chemical reactions lock up almost all of the available carbon. This situation is in contrast with that of $\mathrm{CS}$, for which the abundance shows no clear trend 


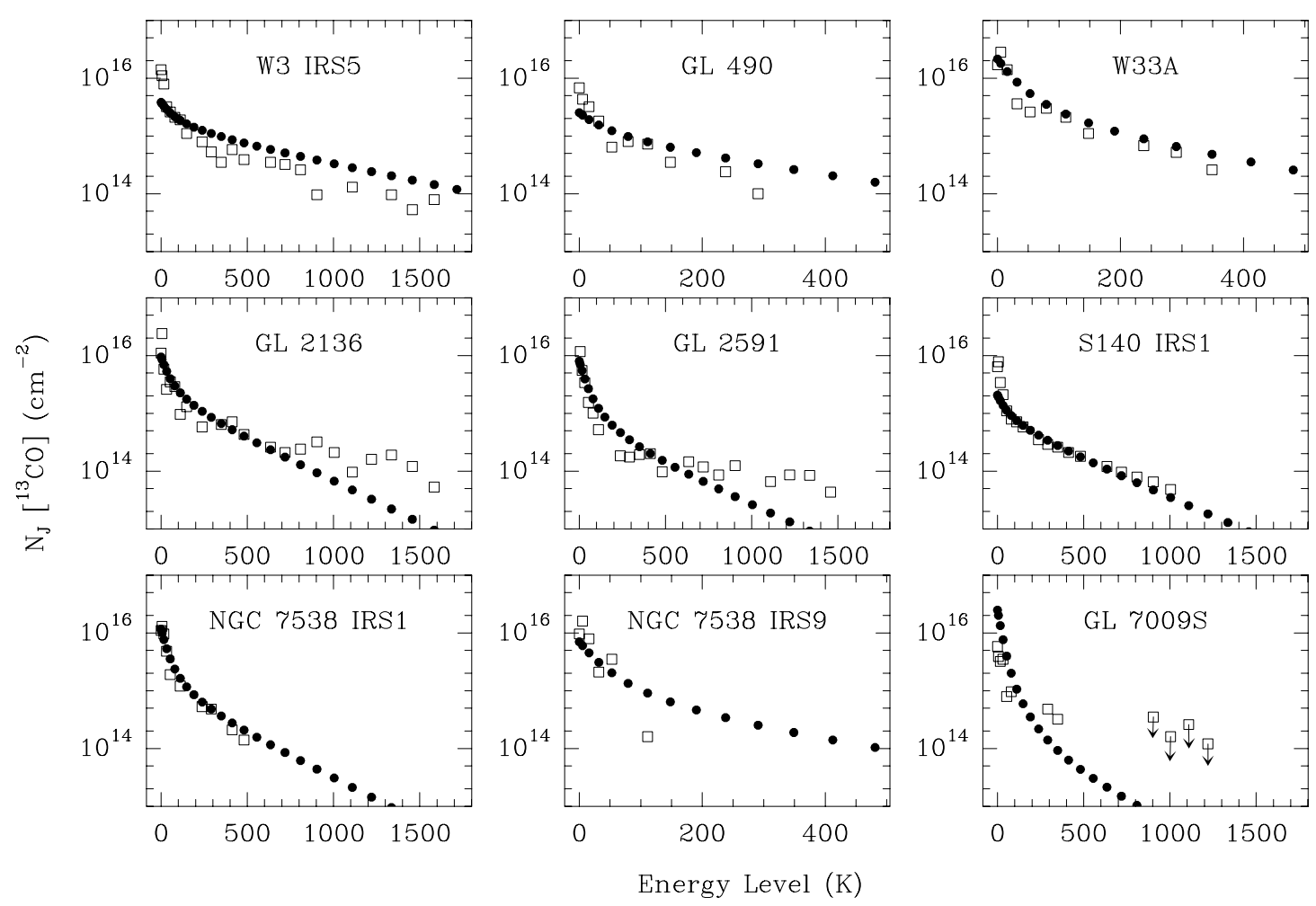

Fig. 9. - Observed and modeled column density in the rotational states of ${ }^{13} \mathrm{CO}$ up to $J=25$ for the NIR-bright sources. Data are from Mitchell et al. 1990, except GL 7009S where they are from Dartois et al. 1998. The model values have been scaled to the observed total column density.

with $\bar{T}$. This difference presumably reflects the much greater chemical inertness of CO compared with that of CS and the lower evaporation temperature of $\mathrm{CO}$ compared with that of CS.

In the case of GL $7009 \mathrm{~S}$, the ${ }^{13} \mathrm{CO}$ column density observed in absorption by Dartois et al. (1998b) is similar to

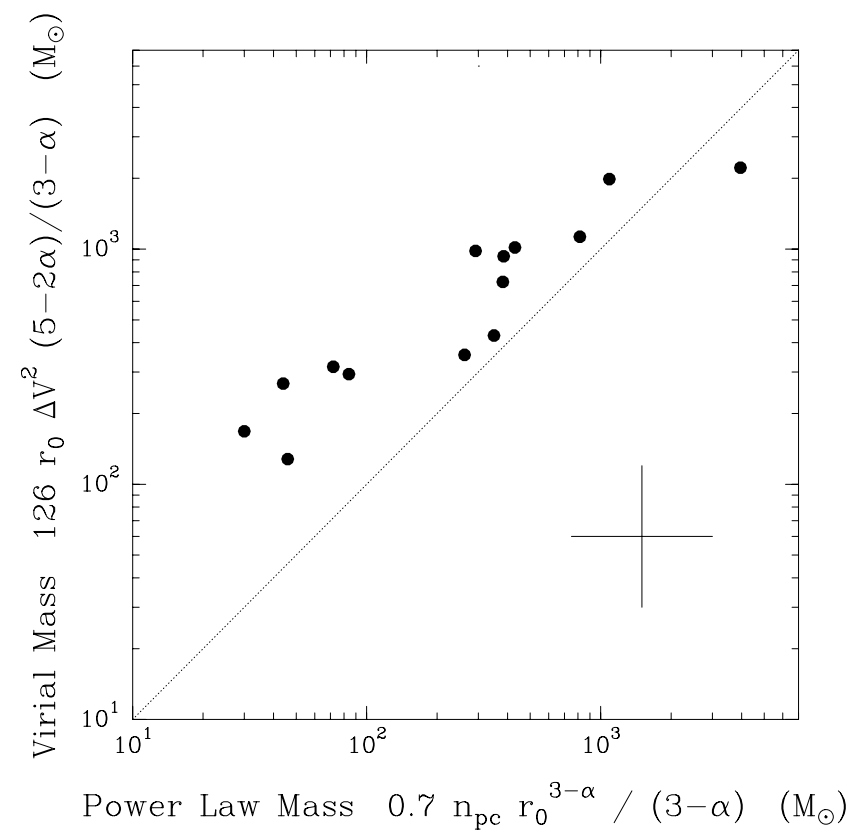

FIG. 10.-Comparison of cloud masses derived from the power-law models with those derived from the virial theorem. The quantity $n_{\mathrm{pc}}$ is the density at a distance of $1 \mathrm{pc}$ from the center; $\Delta V$ is the line width from Table 3 , and the other quantities are in Table 5. that in our model. This implies that along this line of sight, $\mathrm{CO}$ is much less depleted than CS, for which a depletion by a factor $\approx 10$ was found compared with that of the other sources in our sample $(\$ 5.2)$. Again, this can be understood from the difference in evaporation temperature for these two molecules. The large column of CO ice toward, e.g., W3 IRS 5 , is not predicted by our model. We suggest that this

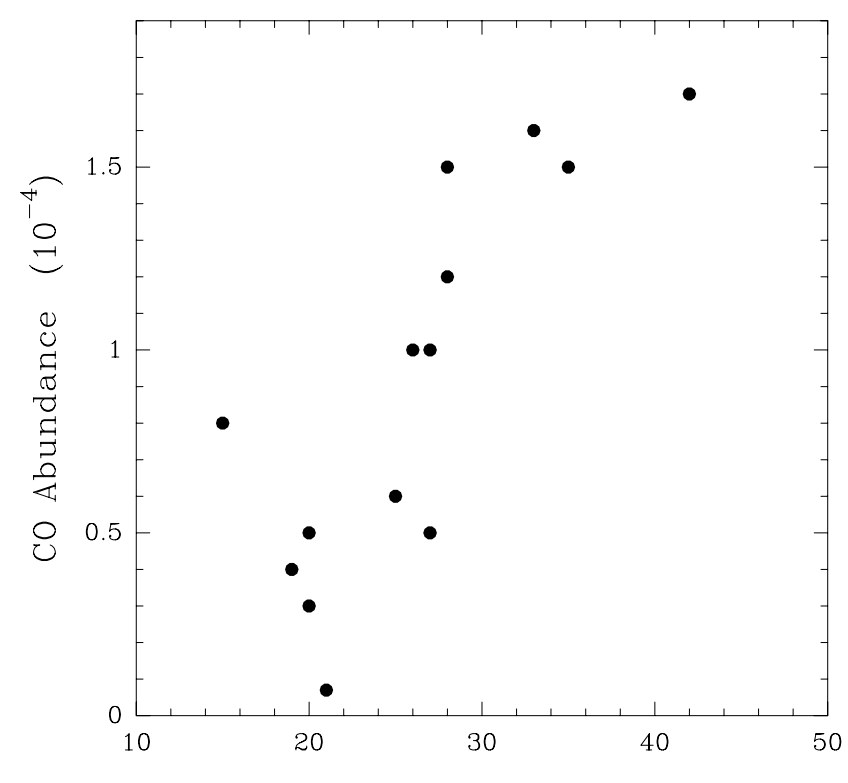

Mass-weighted Temperature (K)

FIG. 11.- Abundance of $\mathrm{CO}$ derived from $\mathrm{C}^{17} \mathrm{O}$ observations plotted against the mass-weighted temperature in our models. 
star-forming core is surrounded by an extended dense cloud that also produces the self-reversed $\mathrm{CO}$ emission profiles. Sources like GL 2591 and GL 2136 do not seem to have such a "skin," or they have at most a translucent one.

Mennella et al. (1998) found that the submillimeter opacity of dust grains increases by $10 \%-50 \%$ when the temperature rises from 24 to $300 \mathrm{~K}$. This effect would decrease the masses of our sources and increase the inferred abundances of $\mathrm{CO}$ and qualitatively produce the same trend as seen in Figure 11. However, over the applicable temperature range, $20-50 \mathrm{~K}$, an effect of only $5 \%-25 \%$ should occur, which is much less than the factor of 3 increase in CO abundance that we find. Although the grain opacity is likely to vary within our sources, they do not influence the conclusion that the $\mathrm{CO}$ abundance is controlled by freezing and sublimation.

\subsection{The Inner $\lesssim 1000 \mathrm{AU}:$ Evidence for Compact Dense Material}

In this section, the envelope models derived in $\S 5$ will be compared to the OVRO continuum data. Since the images presented in Figure 5 show only compact, circularly symmetric structure, a simple one-dimensional analysis in the Fourier plane is sufficient.

The points in Figure 12 are the OVRO data, averaged in annuli around the source in the $u-v$ plane. In the case of W33A, the source MM 2 was subtracted from the data before averaging. The error bars represent the $1 \sigma$ spread between the data points in each bin and do not include the overall calibration error. Superposed on the data points are model curves, derived by calculating the dust emission from the best-fit models (Table 5) and Fourier transforming the result.

Only at the lowest spatial frequencies do the data match the model curves within the calibration error. Most data points lie well above the model curves, and the observed amplitudes do not drop with increasing spatial frequency, as the models do. These differences suggest that the emission detected with OVRO is not related to the envelopes seen in the single-dish data but is caused by the compact structure of size $\lesssim 2$. The same conclusion was reached for GL 2591 by van der Tak et al. (1999).

Toward the highest observed spatial frequencies, 0.5$1 \times 10^{5} \mathrm{rad}^{-1}$, corresponding to $\sim 3000 \mathrm{AU}$ at $1 \mathrm{kpc}$, the compact emission is 10-100 times stronger than that of the envelope model. This factor is a lower limit to the column density contrast between the two components since the compact emission is probably optically thick, as is indicated by the spectral indices. Combined with the envelope column densities in Table 3, this result suggests that the compact emission seen with OVRO can explain the asymmetry observed in the CS line profiles ( $\S 4.1)$.

Further constraints on the nature of the compact emission can be obtained from the flux densities (Table 4), which imply brightness temperatures of $50-80 \mathrm{~K}$ for NGC 7538 IRS 1 and $\approx 1 \mathrm{~K}$ for the other sources. These values imply that the emission is beam diluted by a factor of $\gtrsim 100$ since the physical temperature of the compact structure must be at least that of the surrounding envelope, which acts as an oven (see van der Tak et al. 1999). Assuming a temperature of $200 \mathrm{~K}$ for the compact dust, a lower limit to the mass of $\sim 10 M_{\odot}$ is obtained. In the case of NGC 7538 IRS 1, where free-free and dust emission both contribute at these frequencies (Woody et al. 1989; Akabane et al. 1992), the physical temperature may be greater than $100 \mathrm{~K}$ and the beam dilution correspondingly more. For the other sources, the compact emission is most likely caused by dust, perhaps in the form of a dense shell or of a circumstellar disk. The implied radius of the emitting region, $\lesssim 300-600$ AU at 2-4 $\mathrm{kpc}$, seems small to hide an entire outflow lobe, but, presumably, the dense part of the flow, which emits in high- $J$ CS lines, is confined to its center.

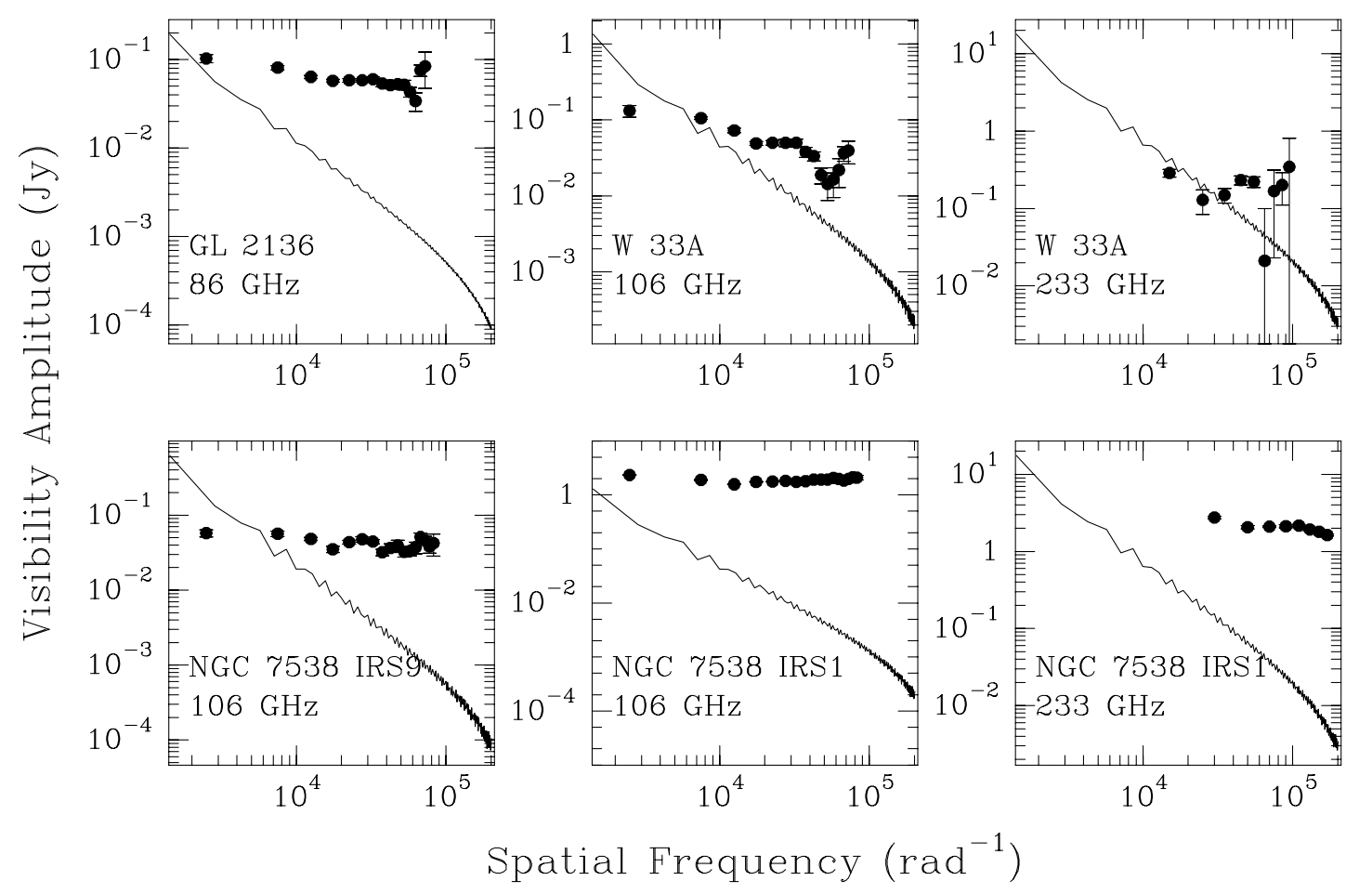

FIG. 12.-Continuum visibilities observed with OVRO compared to the power-law models 


\subsection{Comparison with Low-Mass Objects}

This section discusses possible origins of the spread in $\alpha$ within our sample. First, the value of $\alpha$ may be related to other physical parameters, in particular, luminosity or envelope mass. The structure of the material surrounding pre-main-sequence stars of low ( solar) and intermediate ( 3-8 solar) mass has received considerable attention in the recent literature (e.g., Ladd et al. 1991; Butner et al. 1991; Natta et al. 1993; Butner, Natta, \& Evans 1994; Hogerheijde et al. 1999). These studies generally find $\alpha \approx 2 \pm 0.3$ inside radii of $0.1 \mathrm{pc}$, masses of $\leq 10 M_{\odot}$ and mean densities of $10^{4} \mathrm{~cm}^{-3}$. Of these properties, only the radii are similar to those of the objects studied here; the masses and mean densities are at least 2 orders of magnitude smaller. The density gradients are significantly steeper than those found in this paper, but the gradients for intermediate-mass stars are also lower than those for low-mass stars (Natta et al. 1993). Indeed, in an earlier study of regions of high-mass star formation, Zhou et al. (1994) suggested that more massive star-forming regions tend to have flatter density distributions. However, Figures $13 a$ and $13 b$ show that within our sample, $\alpha$ does not appear correlated with source luminosity or envelope mass. We did not find a relation with any other physical parameters either, such as turbulent pressure as measured by the line width.
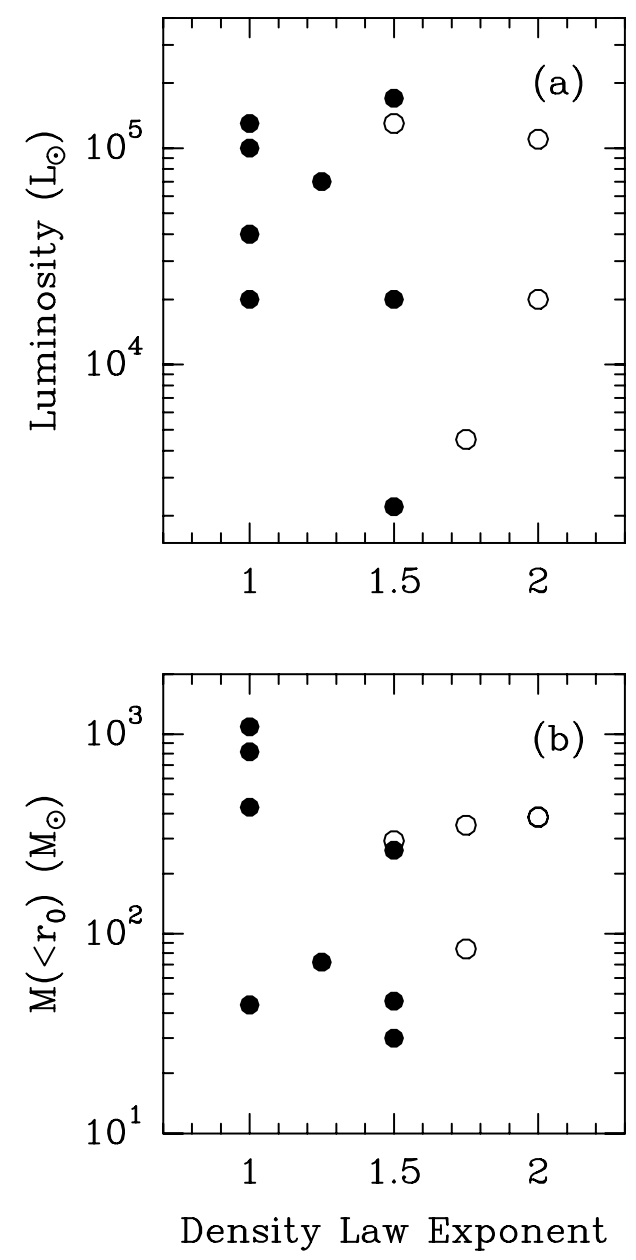

FIG. 13.- Slope of density gradient $\alpha$ vs. source luminosity and mass. Filled symbols indicate the main sample, open symbols the comparison sample. Plotted quantities are explained in the text.
Power laws have been proposed for the density structures of the envelopes of young stars, with the index depending on the dominant term in the pressure. If support against gravitational collapse is caused primarily by thermal pressure, a value of $\alpha=2$ is expected, while if the dominant pressure is of nonthermal origin, the density structure should follow an $\alpha=1$ law. Between these two cases, a continuum of solutions can be constructed (Lizano \& Shu 1989; Myers \& Fuller 1992; McLaughlin \& Pudritz 1997). Our observations may thus indicate that the cores where massive stars form differ from those where low-mass stars form in that they are supported against collapse by a different mechanism. Within this interpretation, the spread in $\alpha$ may be because of source evolution from an $(\alpha=1.0)$ logatrope to a collapsing region $(\alpha=1.5)$.

\subsection{Tracing Envelope Evolution}

Despite having similar radio and infrared properties, our sources show different degrees of dispersal and warming up of their envelopes. This is shown by Boogert et al. (2000) and van Dishoeck (1998) for a source sample similar to ours based on several tracers: the far-infrared color, the fraction of crystalline $\mathrm{CO}_{2}$ ice, the gas/solid ratios of $\mathrm{CO}, \mathrm{CO}_{2}$, and $\mathrm{H}_{2} \mathrm{O}$, and the excitation temperature of $\mathrm{CO}$. These quantities all trace the envelope temperature but all do so in different ways: some trace the dust, others the gas, and some trace small scales and others large scales. As shown by Boogert et al., the temperature indicators correlate with each other. Of particular interest is the fraction of crystalline $\mathrm{CO}_{2}$ ice, because crystallization is an irreversible process, so that this indicator measures the maximum temperature, while the others measure the current temperature. Hence, the temperature variations are not random fluctuations (as in FU Orionis objects) but reflect a systematic increase with time of the temperature of the envelope as a whole.

Figure $14 a$ plots one of these indicators, the far-infrared color, versus the ratio of $M\left(<r_{0}\right)$ to stellar mass, measured as $L^{1 / 3.5}$. This quantity is equivalent to the ratio of submillimeter to bolometric luminosity, which is often used to trace the evolution of young low-mass objects (André et al. 2000). The color $F_{45} / F_{100}$ is the ratio of the flux densities at 45 and $100 \mu \mathrm{m}$, both observed with ISO-LWS in an $80^{\prime \prime}$ beam, which is large enough to cover the entire envelope. These data were provided by A. Boogert (1999, private communication). The plotted quantities are seen to be correlated, with higher temperatures (traced by a larger $\left.F_{45} / F_{100}\right)$ corresponding to lower values of $M\left(<r_{0}\right) / L^{1 / 3.5}$. Thus, the temperature variations are indeed caused by dispersal of the circumstellar material and can be used to trace evolution.

Interestingly, $\alpha$ is not seen to be correlated with farinfrared color (Fig. 14b), and we conclude that on the timescale of the dispersal of the envelopes of massive young stars, the density structure of the envelopes does not change significantly. The masses of the compact sources detected by us and by Woody et al. (1989) and Wyrowski et al. (1999) do not show a relation with $M\left(<r_{0}\right) / L^{1 / 3.5}$ either, although the uncertainty in these masses is rather high.

In addition to $M\left(<r_{0}\right) / L^{1 / 3.5}$, we have considered two "derivative" evolutionary indicators. The first is the bolometric temperature $T_{\text {bol }}$, introduced for low-mass objects by Myers \& Ladd (1993). As the envelopes in those systems are dispersed (probably by the bipolar outflows), their bolo- 

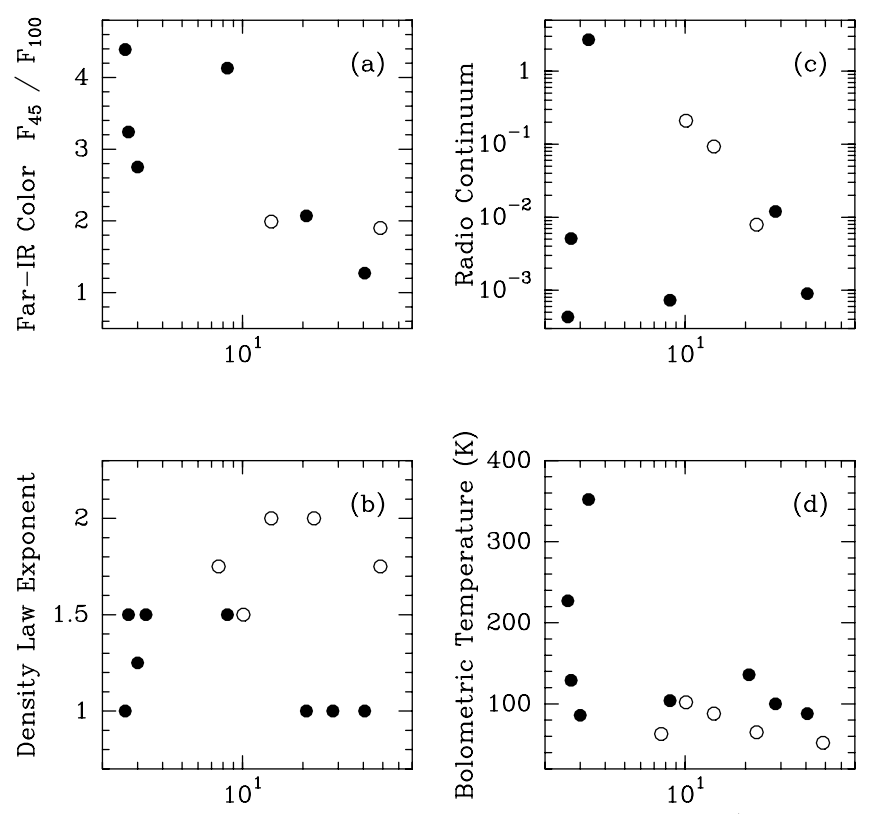

Ratio Envelope to Stellar Mass $\mathrm{M}\left(<\mathrm{r}_{0}\right) / \mathrm{L}^{1 / 3.5}$

FIG. 14.-Possible evolutionary indicators plotted vs. ratio of envelope mass to stellar mass: (a) far-infrared color; (b) slope of density gradient $\alpha$; (c) normalized radio continuum flux density; (d) bolometric temperature. Filled symbols indicate the main sample, open symbols the comparison sample. See text for details.

metric temperatures rise because the far-infrared emission decreases while the near-infrared emission increases (Myers et al. 1998). This process makes $T_{\text {bol }}$ increase monotonically from $\sim 30$ to $3000 \mathrm{~K}$ on a timescale of $10^{6} \mathrm{yr}$. For all but two of our objects, values of $T_{\text {bol }}$ based on the spectral energy distributions of Figure 6 are 50-150 K. This small spread suggests that all the sources are at similar evolutionary stages; $T_{\text {bol }}$ may also work less well than $F_{45} / F_{100}$ because near- and mid-infrared emission have a dependence on source orientation.

Another potential evolutionary indicator is the radio continuum flux, which for a given luminosity and distance is expected to increase with time as the dusty envelope is cleared away and the Lyman continuum photons can all go into ionizing the gas without being absorbed by dust. We have normalized the radio continuum flux densities in Table 1 to the value expected for an $\mathrm{H}$ II region ionized by a main-sequence star of the same luminosity and at the same distance as the source, following Churchwell (1991). However, Figures $14 c$ and $14 d$ show that neither the radio continuum emission nor the bolometric temperature correlates well with $M\left(<r_{0}\right) / L^{1 / 3.5}$, suggesting that these are less useful evolutionary indicators for very young massive objects than $F_{45} / F_{100}$. Possibly the radio emission arises in a wind $(\S 2)$, or the stellar surface temperatures are significantly below main sequence values.

We conclude that dispersal of the envelopes underlies the evolution of both high-mass and low-mass objects but that the observational appearance is very different. The dispersal process can be understood in more detail by recalling that the brightest mid-infrared sources in our sample are the weakest at radio wavelengths and vice versa (Table 1). This anticorrelation suggests that the erosion of the envelope proceeds from the inside out. Massive stars are efficient at removing circumstellar dust by their ionizing UV radiation and by their strong winds. When the innermost, hottest region of dust disappears, the mid-infrared emission will decrease, but the radio continuum will increase because a larger region can be ionized. The compact dust component detected with OVRO is optically thick in the bright midinfrared sources but thin in the others, which trend may also trace dispersal of hot dust. The far-infrared emission will not be affected since it arises on larger scales. These trends are consistent with the properties of our sample, although other processes may play a role as well.

\section{SUMMARY AND CONCLUSIONS}

We have presented maps and spectra of 14 regions of massive star formation in continuum and molecular lines at submillimeter wavelengths. The data are used to develop models of the physical structure of the circumstellar envelopes on $10^{2}-10^{5}$ AU scales. The column density is derived from the submillimeter continuum flux densities, and the temperature structure is calculated self-consistently using the size of the CS emission and the sources' luminosities and distances from the literature. The density structure is constrained with emission lines of $\mathrm{CS}, \mathrm{C}^{34} \mathrm{~S}$, and $\mathrm{H}_{2}$ CO. The following main conclusions are found.

1. The physical structure of the envelopes of deeply embedded massive young stars is characterized by radii of 3-9 $\times 10^{4}$ AU, masses of $10^{2}-10^{3} M_{\odot}$ inside these radii, and visual extinctions of $\sim 10^{2}-10^{3}$ magnitudes. Temperatures increase from $\sim 20 \mathrm{~K}$ at the outer edge to several $100 \mathrm{~K}$ at $\sim 10^{2}$ AU from the star; densities increase from $\sim 10^{4} \mathrm{~cm}^{-3}$ to $\sim 10^{7} \mathrm{~cm}^{-3}$. The slope of the density gradient $\alpha$ is $1.0-1.5$, significantly smaller than the value of $\approx 2$ usually found for low-mass objects. This difference in density structure may be because of nonthermal pressure resisting gravitational collapse, while thermal pressure dominates in lower mass objects. An $\alpha=2$ density structure is found here for two hot cores, but this is an upper limit since the CS abundance may be enhanced in the warm gas close to the star.

2. The shapes of the envelopes deviate somewhat from spherical, as is shown by modeling infrared absorption line data of ${ }^{13} \mathrm{CO}$, the near-infrared continuum, and the CS line profiles. The data are consistent with a decrease of the optical depth by a factor of $\approx 3$ in the central $\lesssim 10^{\prime \prime}$ area. The brightness of our main sample at mid-infrared wavelengths is caused in part by this optical depth effect but also by the flatter density structure. Inhomogeneities on top of the power-law structure are small since the masses obtained by integrating the power-law models agree with masses found from the virial theorem to a factor of 3 . The values of $\alpha$ found from CS are verified by model fits to the maps of dust emission that trace the column density distribution. The only exception is S140 IRS 1, probably because our assumption of a single central heating source is invalid in this case.

3. Modeling of $\mathrm{C}^{17} \mathrm{O}$ emission lines shows that $\approx 40 \%-$ $90 \%$ of the CO gas is depleted onto dust, assuming a dust opacity of $\approx 1 \mathrm{~cm}^{-2} \mathrm{~g}^{-1}$ at $1300 \mu \mathrm{m}$, as found previously for one of our sources, GL 2591 (van der Tak et al. 1999). The derived CO abundances correlate well with the massweighted temperature in our models. This result suggests that in these sources, freeze-out and thermal desorption control the gas-phase abundance of $\mathrm{CO}$. The CS abundance 
is $3 \times 10^{-9}$ on average, ranging from $0.4 \times 10^{-9}$ in the cold source GL $7009 \mathrm{~S}$ to $1-2 \times 10^{-8}$ in the hot-core-type objects.

4. Dense outflowing gas is seen in the $\mathrm{CS}$ and $\mathrm{H}_{2} \mathrm{CO}$ line wings but much more so at blue- than at redshifted velocities. This asymmetry may be caused by absorption by dense, optically thick material within $10^{\prime \prime}$ from the star. The opacity of the power-law models is a factor of $10-100$ below that required for this absorption. However, interferometric continuum observations at 1300-3500 $\mu$ m show compact emission, probably from an 0.3 diameter optically thick dust component. The emission is a factor of $\sim 10-100$ stronger than expected for the envelopes seen in the singledish data and may be because of a dense shell or a circumstellar disk. This component may explain the asymmetric $\mathrm{CS}$ and $\mathrm{H}_{2} \mathrm{CO}$ line profiles.

5. The evolution of these high-mass sources is traced by the overall temperature (measured by the far-infrared color), which increases systematically with the decreasing ratio of envelope mass to stellar mass. The observed anticorrelation of near-infrared and radio continuum emission suggests that the disruption of the envelope proceeds from the inside out. Conventional tracers of the evolution of lowmass objects do not change much over this narrow age range. We conclude that the evolution of high-mass and low-mass envelopes have the same underlying mechanism (envelope dispersal), despite their different observational properties.

The authors are grateful to the staffs of the OVRO, JCMT, CSO, and IRAM $30 \mathrm{~m}$ telescopes-in particular, Remo Tilanus and Fred Baas at the JCMT; Gilles Niccolini at IRAM; and Leonardo Testi, Christine Wilson, and Debra Shepherd at OVRO. We are also indebted to Michiel Hogerheijde for developing the Monte Carlo code, to Göran Sandell for providing the SCUBA maps of NGC 7538 and for his constructive referee report, to Lee Mundy for providing the code to model dust emission profiles, to Yancy Shirley who reduced the SHARC data, to Adwin Boogert for providing the ISO-LWS data, and to Craig Kulesa for sending his CO data. This work also benefited from discussions with Malcolm Walmsley, Ed Churchwell, and Xander Tielens.

This research is supported by NWO grant 614.41.003. G. A. B. gratefully acknowledges support provided by NASA (grants NAG5-2297, -4813). N. J. E. gratefully acknowledges support from the State of Texas and NASA grant NAG5-7203. We also wish to thank the people who maintain the bibliographic databases at CDS (Strasbourg) and ADS (Harvard), of which we have made extensive use.
Acord, J. M., Churchwell, E., \& Wood, D. O. S. 1998, ApJ, 495, L107

Akabane, K., Tsunekawa, S., Inoue, M., Kawabe, R., Ohashi, N., Kameya, O., Ishiguro, M., \& Sofue, Y. 1992, PASJ, 44, 421

Allen, D. A., Hyland, A. R., Longmore, A. J., Caswell, J. L., Goss, W. M., \& Haynes, R. F. 1977, ApJ, 217, 108

Alves, J., Lada, C. J., Lada, E. A., Kenyon, S. J., \& Phelps, R. 1998, ApJ, 506,292

André, P., Ward-Thompson, D., \& Barsony, M. 1993, ApJ, 406, 122

2000, in Protostars and Planets IV, ed. V. Manning, A. Boss, \& S. Russell (Tucson: Univ. of Arizona Press), in press

Anglada, G., Estalella, R., Pastor, J., Rodríguez, L. F., \& Haschick, A. D. 1996, ApJ, 463, 205

Blake, G. A., Sutton, E. C., Masson, C. R., \& Phillips, T. G. 1987, ApJ, 315, 621

Bloomer, J. D., et al. 1998, ApJ, 506, 727

Boogert, A. C. A., et al. 2000, A\&A, 353, 349

Bronfman, L., Nyman, L.-A., \& May, J. 1996, A\&AS, 115, 81

Butner, H. M., Evans, N. J., II, Lester, D. F., Levreault, R. M., \& Strom, S. E. 1991, ApJ, 376, 636

Butner, H. M., Natta, A., \& Evans, N. J., II. 1994, ApJ, 420, 326

Cabrit, S., Raga, A., \& Gueth, F. 1997, in IAU Symp. 182, Herbig-Haro Flows and the Birth of Low-Mass Stars, ed. B. Reipurth \& C. Bertout (Dordrecht: Kluwer), 163

Campbell, B. 1984, ApJ, 287, 334

Campbell, M. F., Butner, H. M., Harvey, P. M., Evans, N. J., II, Campbell, M. B., \& Sabbey, C. N. 1995, ApJ, 454, 831

Capps, R. W., Gillett, F. C., \& Knacke, R. F. 1978, ApJ, 226, 863

Carpenter, J. M., Snell, R. L., Schloerb, F. P., \& Skrutskie, M. F. 1993, ApJ, 407,657

Carr, J. S., Evans, N. J., II, Lacy, J. H., \& Zhou, S. 1995, ApJ, 450, 667

Cesaroni, R., Felli, M., Testi, L., Walmsley, C., \& Olmi, L. 1997, A\&A, 325, 725

Cesaroni, R., Felli, M., Jenness, T., Neri, R., Olmi, L., Robberto, M., Testi, L., \& Walmsley, C. M. 1999, A\&A, 345, 949

Cesaroni, R., Hofner, P., Walmsley, C. M., \& Churchwell, E. 1999, A\&A, 331,709

Chandler, C. J., Gear, W. K., \& Chini, R. 1993, MNRAS, 260, 337

Charnley, S. B., Tielens, A. G. G. M., \& Millar, T. J. 1992, ApJ, 399, L71

Cheung, L. H., Frogel, J. A., Hauser, M. G., \& Gezari, D. Y. 1980, ApJ, 240, 74

Chini, R., Henning, T., \& Pfau, W. 1991, A\&A, 247, 157

Chini, R., Krügel, E., \& Kreysa, E. 1986, A\&A, 167, 315

Churchwell, E., Wolfire, M. G., \& Wood, D. O. S. 1990, ApJ, 354, 247

Churchwell, E. B. 1991, in The Physics of Star Formation and Early Stellar Evolution, ed. C. J. Lada \& N. D. Kylafis (Dordrecht: Kluwer), 221 .1999, in The Origin of Stars and Planetary Systems, ed. C. J. Lada $\&$ N. D. Kylafis (Dordrecht: Kluwer), 515

Claussen, M. J., Gaume, R. A., Johnston, K. J., \& Wilson, T. L. 1994, ApJ, 424, L41
Colley, D. 1980, MNRAS, 193, 495

Crampton, D., \& Fisher, W. A. 1974, Publ. Dom. Astrophys. Obs. Victoria, 14,283

Crampton, D., Georgelin, Y. M., \& Georgelin, Y. P. 1978, A\&A, 66, 1

Dartois, E. 1998, Ph.D. thesis, Université de Paris VI

Dartois, E., et al. 1998a, A\&A, 338, L21

Dartois, E., d'Hendecourt, L., Boulanger, F., Jourdain De Muizon, M., Breitfellner, M., Puget, J. L., \& Habing, H. J. 1998b, A\&A, 331, 651

d'Hendecourt, L., et al. 1996, A\&A, 315, L365

Dyck, H. M., \& Simon, T. 1977, ApJ, 211, 421

Egan, M. P., Leung, C. M., \& Spagna, G. R. J. 1988, Comput. Phys. Commun., 48, 271

Evans, N. J., II, Beckwith, S., Brown, R. L., \& Gilmore, W. 1979, ApJ, 227, 450

Evans, N. J., II. 1999, ARA\&A, 37, 311

Evans, N. J., II, Mundy, L. G., Kutner, M. L., \& Depoy, D. L. 1989, ApJ, 346,212

Faison, M., Churchwell, E., Hofner, P., Hackwell, J., Lynch, D. K., \& Russell, R. W. 1998, ApJ, 500, 280

Garay, G., \& Lizano, S. 1999, PASP, 111, 1049

Green, S. 1991, ApJS, 76, 979

Gürtler, J., Henning, T., Krügel, E., \& Chini, R. 1991, A\&A, 252, 801

Harvey, P. M., Campbell, M. F., \& Hoffmann, W. F. 1977, ApJ, 211, 786

Harvey, P. M., Campbell, M. F., Hoffmann, W. F., Thronson, H. A., J., \& Gatley, I. 1979, ApJ, 229, 990

Harvey, P. M., \& Gatley, I. 1983, ApJ, 269, 613

Harvey, P. M., Joy, M., Lester, D. F., \& Wilking, B. A. 1986, ApJ, 300, 737

Harvey, P. M., Lester, D. F., Colome, C., Smith, B., Monin, J.-L., \& Vauglin, I. 1994, ApJ, 433, 187

Hatchell, J., Fuller, G. A., Millar, T. J., Thompson, M. A., \& Macdonald, G. H. 2000, A\&A, in press

Hauschildt, H., Gusten, R., Phillips, T., Schilke, P., Serabyn, E., \& Walker, C. 1993, A\&A, 273, L23

Helmich, F. P., Jansen, D. J., de Graauw, T., Groesbeck, T. D., \& van Dishoeck, E. F. 1994, A\&A, 283, 626

Helmich, F. P., \& van Dishoeck, E. F. 1997, A\&AS, 124, 205

Henkel, C., Wilson, T. L., \& Johnston, K. J. 1984, ApJ, 282, L93

Henning, T. 1997, in IAU Symp. 178, Molecules in Astrophysics: Probes and Processes, ed. E. F. van Dishoeck (Dordrecht: Kluwer), 343

Hodapp, K.-W. 1994, ApJS, 94, 615

Hogerheijde, M. R. \& Sandell, G. 2000, ApJ, 534, 880

Hogerheijde, M. R., van Dishoeck, E. F., Salverda, J. M., \& Blake, G. A. 1999, ApJ, 513, 350

Humphreys, R. M. 1978, ApJS, 38, 309

Hunter, T. R. 1998, PASP, 110, 634

Hunter, T. R., Benford, D. J., \& Serabyn, E. 1996, PASP, 108, 1042

Jaffe, D. T., Hildebrand, R. H., Keene, J., Harper, D. A., Loewenstein, R. F., \& Moran, J. M. 1984, ApJ, 281, 225

Jansen, D. J., van Dishoeck, E. F., \& Black, J. H. 1994, A\&A, 282, 605 
Johnston, K. J., Henkel, C., \& Wilson, T. L. 1984, ApJ, 285, L85

Kastner, J. H., Weintraub, D. A., Snell, R. L., Sandell, G., Aspin, C., Hughes, D. H., \& Baas, F. 1994, ApJ, 425, 695

Keto, E., Proctor, D., Ball, R., Arens, J., \& Jernigan, G. 1992, ApJ, 401, L113

Kurtz, S., Cesaroni, R., Churchwell, E., Hofner, P., \& Walmsley, M. 2000, in Protostars and Planets IV, ed. V. Manning, A. Boss, \& S. Russell (Tucson: Univ. of Arizona Press), in press

Kurtz, S., Churchwell, E., \& Wood, D. O. S. 1994, ApJS, 91, 659

Lacy, J. H., Knacke, R., Geballe, T. R., \& Tokunaga, A. T. 1994, ApJ, 428, L69

Lada, C. J., Thronson, H. A., J., Smith, H. A., Schwartz, P. R., \& Glaccum, W. 1984, ApJ, 286, 302

Ladd, E. F., Adams, F. C., Fuller, G. A., Myers, P. C., Casey, S., Davidson, J. A., Harper, D. A., \& Padman, R. 1991, ApJ, 382, 555

Ladd, E. F., Deane, J. R., Sanders, D. B., \& Wynn-Williams, C. G. 1993, ApJ, 419, 186

Larson, R. B. 1969, MNRAS, 145, 271

Lebofsky, M. J., Kleinmann, S. G., Rieke, G. H., \& Low, F. J. 1976, ApJ, 206, L157

Lester, D. F., Harvey, P. M., Joy, M., \& Ellis, H. B. J. 1986, ApJ, 309, 80

Lightfoot, J. F., Deighton, D. W., Furniss, I., Glencross, W. M., Hirst, C. J., Jennings, R. E., \& Poulter, G. 1984, MNRAS, 208, 197

Lis, D. C., Serabyn, E., Keene, J., Dowell, C. D., Benford, D. J., Phillips, T. G., Hunter, T. R., \& Wang, N. 1998, ApJ, 509, 299

Lizano, S., \& Shu, F. H. 1989, ApJ, 342, 834

Luhman, K. L., Rieke, G. H., Lada, C. J., \& Lada, E. A. 1998, ApJ, 508, 347

Mangum, J. G., \& Wootten, A. 1993, ApJS, 89, 123

McCutcheon, W. H., Sato, T., Purton, C. R., Matthews, H. E., \& Dewdney, P. E. 1995, AJ, 110, 1762

McLaughlin, D. E., \& Pudritz, R. E. 1996, ApJ, 469, 194

. 1997, ApJ, 476, 750

Megeath, S., Herter, T., Beichman, C., Gautier, N., Hester, J., Rayner, J., \& Shupe, D. 1996, A\&A, 307, 775

Mennella, V., Brucato, J. R., Colangeli, L., Palumbo, P., Rotundi, A., \& Bussoletti, E. 1998, ApJ, 496, 1058

Mitchell, G. F., Maillard, J.-P., Allen, M., Beer, R., \& Belcourt, K. 1990, ApJ, 363, 554

Mitchell, G. F., Maillard, J. P., \& Hasegawa, T. I. 1991, ApJ, 371, 342

Moorwood, A. F. M., \& Salinari, P. 1981, A\&A, 94, 299

Mundy, L. G., Looney, L. W., \& Welch, W. J. 2000, in Protostars and Planets IV, ed. V. Manning, A. Boss, \& S. Russell (Tucson: Univ. of Arizona Press), in press

Myers, P. C., Adams, F. C., Chen, H., \& Schaff, E. 1998, ApJ, 492, 703

Myers, P. C., \& Fuller, G. A. 1992, ApJ, 396, 631

Myers, P. C., \& Ladd, E. F. 1993, ApJ, 413, L47

Natta, A., Palla, F., Butner, H. M., Evans, N. J. , II, \& Harvey, P. M. 1993, ApJ, 406, 674

Neckel, T. 1978, A\&A, 69, 51

Olmi, L., \& Cesaroni, R. 1999, A\&A, 352, 266

Ossenkopf, V., \& Henning, T. 1994, A\&A, 291, 943

Padin, S., et al. 1989, ApJ, 337, L45

Padin, S., Scott, S. L., Woody, D. P., Scoville, N. Z., Seling, T. V., Finch, R. P., Giovanine, C. J., \& Lawrence, R. P. 1991, PASP, 103, 461

Plume, R., Jaffe, D. T., \& Evans, N.J., II. 1992, ApJS, 78, 505

Plume, R., Jaffe, D. T., Evans, N.J., II, Martín-Pintado, J., \& GomézGonzález, J. 1997, ApJ, 476, 730

Plume, R., Jaffe, D. T., \& Keene, J. 1994, ApJ, 425, L49

Pratap, P., Snyder, L. E., \& Batrla, W. 1992, ApJ, 387, 241
Reid, M. J., Argon, A. L., Masson, C. R., Menten, K. M., \& Moran, J. M. 1995, ApJ, 443, 238

Rengarajan, T. N., \& Ho, P. T. P. 1996, ApJ, 465, 363

Richardson, K. J., Sandell, G., \& Krisciunas, K. 1989, A\&A, 224, 199

Rodríguez, L. F., Canto, J., \& Moran, J. M. 1982, ApJ, 255, 103

Sandell, G. 1994, MNRAS, 271, 75

Sandford, S. A., \& Allamandola, L. J. 1993, ApJ, 417, 815

Shu, F., Najita, J., Galli, D., Ostriker, E., \& Lizano, S. 1993, in Protostars and Planets III, ed. E. H. Levy \& J. Lunine (Tucson: Univ. of Arizona Press), 3

Shu, F. H. 1977, ApJ, 214, 488

Simon, M., Felli, M., Massi, M., Cassar, L., \& Fischer, J. 1983, ApJ, 266, 623

Snell, R. L., Scoville, N. Z., Sanders, D. B., \& Erickson, N. R. 1984, ApJ, 284,176

Spaans, M., \& van Dishoeck, E. F. 1997, A\&A, 323, 953

Sridharan, T. K., Menten, K. M., Wyrowski, F., \& Schilke, P. 1999, in Proc. Star Formation 1999, ed. T. Nakamoto (Nagoya, Japan: Nobeyama Radio Observatory), 183

Stier, M. T., Jaffe, D. T., Rengarajan, T. N., Fazio, G. G., Maxson, C. W., McBreen, B., Loughran, L., Serio, S., \& Sciortino, S. 1984, ApJ, 283, 573

Straw, S. M., \& Hyland, A. R. 1989, ApJ, 340, 318

Thronson, H. A. J., \& Harper, D. A. 1979, ApJ, 230, 133

Thum, C., \& Lemke, D. 1975, A\&A, 41, 467

Tieftrunk, A., Gaume, R., Claussen, M., Wilson, T., \& Johnston, K. 1997, A\&A, 318, 931

Tofani, G., Felli, M., Taylor, G., \& Hunter, T. 1995, A\&AS, 112, 299

Turner, B. E., Chan, K. W., Green, S., \& Lubowich, D. A. 1992, ApJ, 399, 114

Turner, J. L., \& Welch, W. J. 1984, ApJ, 287, L81

van der Tak, F. F. S., van Dishoeck, E. F., Evans, N. J., II, Bakker, E. J., \& Blake, G. A. 1999, ApJ, 522, 991

van Dishoeck, E. F. 1998, in Discuss. Faraday Soc., 109, 31

van Dishoeck, E. F., \& Blake, G. A. 1998, ARA\&A, 36, 317

van Dishoeck, E. F., et al. 1998, in ASP Conf. Proc. 132, Star Formation with the Infrared Space Observatory, ed. J. L. Yun \& R. Liseau (San Francisco: ASP), 54

Visser, A. E., Richer, J. S., Chandler, C. J., \& Padman, R. 1998, MNRAS, 301,585

Walmsley, M. 1995, Rev. Mexicana de Astron. Astrofis. Ser. de Conf., 1, 137

Wang, Y., Jaffe, D. T., Evans, N. J., II, Hayashi, M., Tatematsu, K., \& Zhou, S. 1993, ApJ, 419, 707

Werner, M. W., Becklin, E. E., Gatley, I., Matthews, K., Neugebauer, G., \& Wynn-Williams, C. G. 1979, MNRAS, 188, 463

Wilking, B. A., Blackwell, J. H., \& Mundy, L. G. 1990, AJ, 100, 758

Willner, S. P., et al. 1982, ApJ, 253, 174

Wilson, T. L., \& Rood, R. 1994, ARA\&A, 32, 191

Wood, D. O. S., \& Churchwell, E. 1989, ApJS, 69, 831

Woody, D. P., Scott, S. L., Scoville, N. Z., Mundy, L. G., Sargent, A. I., Padin, S., Tinney, C. G., \& Wilson, C. D. 1989, ApJ, 337, L41

Wynn-Williams, C. G., Becklin, E. E., \& Neugebauer, G. 1972, MNRAS, 160,1

Wyrowski, F., Schilke, P., Walmsley, C. M., \& Menten, K. M. 1999, ApJ, $514, \mathrm{~L} 43$

Zhou, S., Butner, H. M., Evans, N. J., II, Güsten, R., Kutner, M. L., \& Mundy, L. G. 1994, ApJ, 428, 219

Zhou, S., Evans, N. J., II, Güsten, R., Mundy, L. G., \& Kutner, M. L. 1991, ApJ, 372, 518 